\title{
NEW FORMULATION FOR ESTIMATING THE DAMPING PARAMETER OF THE KELVIN-VOIGT MODEL FOR SEISMIC POUNDING SIMULATION
}

\author{
F. López-Almansa ${ }^{* 1}$, A. Kharazian ${ }^{2}$ \\ ${ }^{1}$ Department of Architecture Technology, Technical University of Catalonia, Avda. Diagonal 649, 08028 Barcelona, francesc.lopez- \\ almansa@upc.edu. Currently: Associate Researcher RiNA, Institute of Civil Works, Austral University of Chile, Valdivia. \\ ${ }^{2}$ Department of Civil and Environmental Engineering, Technical University of Catalonia, Campus Nord UPC, 08034 Barcelona, \\ alireza.kharazian@upc.edu \\ (*) Corresponding author: Francisco López Almansa
}

\begin{abstract}
Seismic pounding between adjoining buildings frequently causes serious damage; although collision can be avoided with proper separation, can still occur due to code non-fulfillment, loose requirements of old codes, and seismicity underestimation. Inside this context, this work deals with collision between two buildings with aligned slabs. The simulation of this phenomenon is not obvious, involving stress traveling waves, high-frequency behavior, and local effects. Complex distributed continuum mechanics-based models can be used, but are timeconsuming; conversely, the concentrated Kelvin-Voigt model can be utilized instead, being simple and inexpensive, yet accurate. Its behavior is characterized by damping and stiffness parameters; the damping influence is particularly important and a number of estimation criteria have been proposed. Among them, the Anagnostopoulos formulation is simple, and provides satisfactory results in most situations. That formulation consists in estimating the damping parameter after a given target value of the coefficient of restitution; the influence, during impact, of the colliding building structures and the seismic excitation is neglected. This paper proposes an alternative approach that releases one of the aforementioned assumptions: the influence of the building structures and their initial separation is taken into consideration. A simplified parametric study oriented to investigate the performance of the proposed strategy is performed; it is found that the accuracy of the Anagnostopoulos formulation is improved in a number of situations. Noticeably, this gain is obtained at a low computational cost. The proposed formulation is satisfactorily utilized to analyze pounding between two multistory multi-bay RC buildings and to simulate a shaking table pounding experiment.
\end{abstract}

Keywords: Seismic pounding; colliding adjoining buildings; Kelvin-Voigt model; numerical simulation; damping parameter estimation

\section{INTRODUCTION}

Impact between contiguous buildings under strong seismic events is a relevant issue since the huge forces that are generated during the collision significantly affect the dynamic behavior of the pounding buildings. On some occasions, the effect of impact might be beneficial, mainly in terms of inter-story drift; conversely, in many other situations, pounding is detrimental, particularly in terms of absolute acceleration. Collapses and structural and nonstructural damage of buildings due to seismic pounding have been reported [1-9]. Although such collision can be avoided by adequately separating the involved buildings, and this gap is routinely required by the design codes, impact can anyway occur because of several reasons: sometimes code prescriptions are not fulfilled, some past codes did not oblige any such separation, and the seismicity can be underestimated. Therefore, seismic pounding of buildings is something to be taken into consideration.

Collision between adjoining buildings can be classified into two categories: slab-to-slab and slab-to-column (or slab-to-wall) impact; they correspond to aligned and unaligned slabs, respectively. The second type is by far more dangerous, since the impact of a rigid and massive slab on a column (or even on a wall) is most likely to lead to collapse. On the other hand, the first type is not free of danger, and is considerably more frequent, since adjoining buildings with unaligned slabs are regularly avoided. Moreover, the numerical simulation of slab-to-slab impact is highly challenging, as discussed later. Thus, this study is focused on seismic pounding of adjoining buildings with aligned slabs.

As outlined in the previous paragraph, collision between two building slabs is a complex phenomenon, because it 
involves stress traveling waves, high-frequency behavior, and significant local effects [10, 11]. Certainly, sophisticated mechanics-based numerical models are available, but they are highly time-consuming. On the other hand, the 3-D continuum partial derivative equations of motion (distributed-parameter models) can be solved exactly in some geometrically simple cases, but the ensuing closed-form solutions can be useful only when the required simplifying assumptions are reasonable. Given these circumstances, the description of the analyzed type of impact with concentrated models has been suggested [12]. The most simple and spread one is the linear viscoelastic Kelvin-Voigt model [13], consisting in the parallel combination of a spring and a dash-pot, together with the consideration of the gap between the colliding slabs, as described later in Figure 1.

The Kelvin-Voigt model is simple, robust and computationally inexpensive, providing reasonable accuracy; moreover, it is implemented in the most common software codes (ETABS, SeismoStruct, OpenSees, among others). On the other hand, sound criteria for selecting the damping parameter are available [14]; with regard to the stiffness parameter, it has proven to be less relevant. Conversely to these positive issues, some studies $[15,16]$ have pointed out that the Kelvin-Voigt model exhibits some degree of inconsistency, since the contact force can take negative values, despite this model being compression-only. With the aim of fixing this conflict, the works [17-19] depict modifications of the Kelvin-Voigt model; however, the study [16] demonstrates that their results are similar to those of the normal Kelvin-Voigt model. In addition, other models such as Hertzdamp [15], nonlinear viscoelastic [20], and Hunt-Crossley [21] have been proposed. These models are computationally more expensive and less robust, are not implemented in the major software codes, and no comprehensive studies providing criteria for selecting the values of the parameters have been reported. Also, the aforementioned inconsistency in the Kelvin-Voigt model does not affect its overall performance [22]. Therefore, the Kelvin-Voigt model is a valid and practical tool for global studies on seismic pounding between buildings with aligned slabs.

As stated in the previous paragraph, criteria for estimating the damping parameter of Kelvin-Voigt model are well established. According to [14], the damping parameter is selected after a simple closed-form expression providing the damping in terms of a target value of the coefficient of restitution; this expression is derived by neglecting, during the impact duration, the influence of the colliding building structures and of the seismic excitation. This approach has proven basically satisfactory $[13,16,22,23]$, but further improvement is still possible.

As discussed previously, less attention has been paid to the stiffness parameter, since it has no intense effect on the buildings response. More precisely, the effect on post-impact velocity, drift displacement, and story shear force is weak [13]; conversely, impact force and absolute acceleration are more sensitive to the stiffness of the KelvinVoigt model [11]. Given these considerations and the fact that a considerable number of sound criteria to select the stiffness parameter have been reported [12, 13, 22, 24, 25], this research is focused on the damping parameter.

This paper presents a new formulation where the damping parameter of the Kelvin-Voigt model is selected, like in the previous approach [14], after the target coefficient of restitution. The proposed formulation accounts, during the impact duration, for the influence of the stiffness and damping of the colliding buildings; the effect of input is disregarded, because such repercussion is random and has proven limited in all the analyzed situations. The proposed formulation leads to a simple algorithm. Their required input parameters depend on the building structural properties and on the velocities at the beginning of impact; criteria for selecting such velocities are provided. A simplified parametrical study on two single-story single-bay frames is carried out; its objective is to investigate the performance of the proposed formulation, mainly in comparison with the previous one [14]. Numerical examples on pounding between pairs of multi-story multi-bay RC frames under seismic excitation are presented; the obtained results highlight the capacity of the proposed approach and, in some cases, their higher performance compared to the previous one. These remarks are further emphasized in the numerical simulation of shaking table pounding experiments on pairs of RC colliding frames.

Parametrical studies on seismic pounding between pairs of three-to-five story adjoining RC buildings with aligned slabs using the new formulation are being performed [11]. The final objective of this long-term research is to provide simple and efficient simulation tools and to study the relevance of seismic pounding in the most common situations.

\section{KELVIN-VOIGT MODEL OF POUNDING}

\subsection{Model description}


The Kelvin-Voigt model is a zero-length link element consisting of a linear spring and a linear dash-pot in parallel, as displayed in Figure 1.b. In Figure 1.b, $m_{1}$ and $m_{\mathrm{r}}$ are the equivalent masses of the left and right colliding slabs, $d$ is the gap size, and $k$ and $c$ are the stiffness and damping parameters of the Kelvin-Voigt model, respectively. Noticeably, the masses $m_{1}$ and $m_{\mathrm{r}}$ are not actually a part of the model; they have been included herein for further clarity. In Figure 1.b, $v_{1}$ and $v_{\mathrm{r}}$ are the colliding sections velocities of the left and right buildings, respectively; $x_{1}$ and $x_{\mathrm{r}}$ are the corresponding coordinates $\left(v_{1}=\dot{x}_{1}\right.$ and $\left.v_{\mathrm{r}}=\dot{x}_{\mathrm{r}}\right)$. Figure 1.c represents a strained state of the KelvinVoigt model; noticeably, if $x_{1}-x_{\mathrm{r}}>d$, there is relative deformation between both slabs. Figure 1.a displays the distribution of the Kelvin-Voigt models in a pair of adjoining pounding buildings.

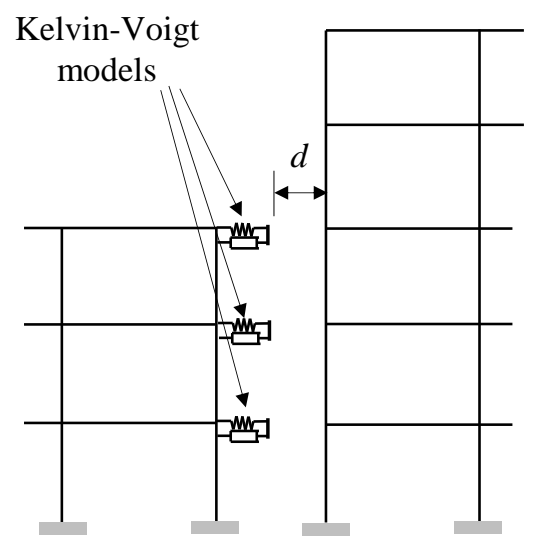

(a) Distribution of Kelvin-Voigt models

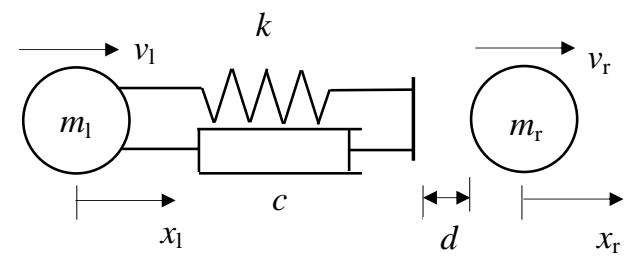

(b) Unstrained Kelvin-Voigt model

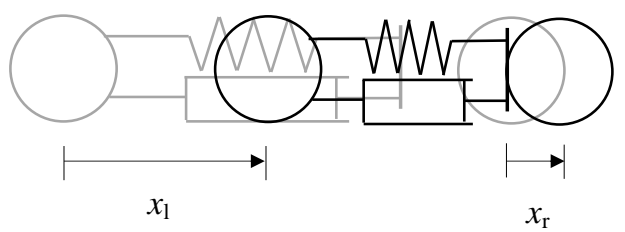

(c) Strained Kelvin-Voigt model during impact

Figure 1. Lumped Kelvin-Voigt models for simulation of pounding between adjoining buildings with aligned slabs

The constitutive law of the Kelvin-Voigt model is given by

$$
F=k\left(x_{1}-x_{\mathrm{r}}-d\right)+c\left(\dot{x}_{1}-\dot{x}_{\mathrm{r}}\right) \text { if } x_{\mathrm{l}}-x_{\mathrm{r}}>d
$$

$$
F=0 \text { if } x_{1}-x_{\mathrm{r}} \leq d
$$

\subsection{Previously proposed criteria for estimating the stiffness parameter}

As discussed in the Introduction, in past studies [12, 13, 17, 22, 24, 26, 27], the Kelvin-Voigt model stiffness has been considered to have little influence on the displacement response, and be strongly dependent on many highly and randomly varying parameters [13]. Therefore, only loose criteria for selecting such parameter have been provided. The conclusions of studies $[13,22]$ suggest using significantly higher values than the lateral stiffness of the colliding buildings, [26] recommends that the local periods of the mass-impact springs keep below the lowest translational periods of the pounding buildings, [24] proposes considering the axial stiffness of the floor slabs, [12] also refers to the axial stiffness of the slabs but checks as well higher and lower values, and finally, [25] proposes an expression depending on the Hertz stiffness for spherical contact, and on the maximum penetration. In any case, the stiffness of the Kelvin-Voigt model is considerably higher than the lateral stiffness of the buildings.

Noticeably, in the allegedly more accurate distributed parameter formulation [11], the impact force is constant during the collision duration, while in the concentrated Kelvin-Voigt model such force is given by equation (1). Therefore, it is not possible to obtain any exact equivalence between both formulations. This fact had been already pointed out in [10].

\subsection{Previously proposed criterion for estimating the damping parameter}

Conversely to stiffness, damping is universally recognized as a more relevant parameter, significantly affecting virtually all the relevant response parameters (drift displacement, story shear force, absolute acceleration, impact force, absorbed energy, etc.); therefore, more attention has been paid to this issue. The study [14] derives a closedform expression of damping in terms of the coefficient of restitution $r$. Such coefficient is defined as the ratio between the post-impact and initial relative velocities between the colliding masses: 


$$
r=\frac{v_{\mathrm{r}}^{\prime}-v_{1}^{\prime}}{v_{1}-v_{\mathrm{r}}}
$$

Equation (2) shows that $r$ ranges between 0 (plastic impact) and 1 (elastic impact). The coefficient of restitution has a more recognizable physical meaning than the damping parameter; therefore, commonly it is preferred to start the process by selecting a target value $r_{\mathrm{T}}$.

The work [14] neglects, during the impact, the influences of the colliding building structures and the seismic excitation. These assumptions are based on the higher stiffness and damping values used in the Kelvin-Voigt model compared to the buildings, and on the high input randomness, given the highly short impact duration. After these simplifications, Anagnostopoulos [14] performed a modal analysis of the ensuing two-degree of freedom system (Figure 1.b) and derived closed-form solutions of the uncoupled scalar equations of motion in modal coordinates. The first mode involves only rigid-body motion and has no stiffness and no damping; regarding the second mode, its natural frequency $(\omega)$ and damping ratio $(\zeta)$ are given by

$$
\omega=\sqrt{k \frac{m_{1}+m_{\mathrm{r}}}{m_{\mathrm{l}} m_{\mathrm{r}}}} \quad \zeta=\frac{c}{2} \sqrt{\frac{m_{1}+m_{\mathrm{r}}}{k m_{1} m_{\mathrm{r}}}}
$$

The aforementioned closed-form solution of the second mode is determined after their parameters in equation (3). Then, the impact duration $\left(t_{\mathrm{imp}}\right)$ is obtained as $t_{\mathrm{imp}}=\frac{\pi}{\omega\left(1-\zeta^{2}\right)^{1 / 2}}$, and later the target coefficient of restitution is determined as:

$$
r_{\mathrm{T}}=e^{\frac{-\zeta_{\mathrm{A}} \pi}{\left(1-\zeta_{\mathrm{A}}^{2}\right)^{1 / 2}}}
$$

In this equation, the subindex $\mathrm{A}$ accounts for Anagnostopoulos. This expression can be inverted to provide the damping ratio in terms of the coefficient of restitution: $\zeta_{\mathrm{A}}=-\ln r_{\mathrm{T}} /\left(\pi^{2}+\ln ^{2} r_{\mathrm{T}}\right)^{1 / 2}$. Equation (4) shows that, if $r_{\mathrm{T}}=$ $0, \zeta_{\mathrm{A}}=1$ and if $r_{\mathrm{T}}=1, \zeta_{\mathrm{A}}=0$. Noticeably, in intermediate cases corresponding to realistic situations, $\zeta_{\mathrm{A}}$ tend to be significantly smaller than 1 ; for instance, if $r_{\mathrm{T}}=0.7, \zeta_{\mathrm{A}}=0.1128$ and if $r_{\mathrm{T}}=0.5, \zeta_{\mathrm{A}}=0.2155$.

To summarize, this approach consists in estimating initially a target value $r_{\mathrm{T}}$ of the coefficient of restitution, obtaining then the damping ratio $\zeta_{\mathrm{A}}$ with equation (4), and finally determining the damping parameter $c_{\mathrm{A}}$ with the second equation (3) $\left(c_{\mathrm{A}}=2 \zeta_{\mathrm{A}} \sqrt{\frac{k m_{1} m_{\mathrm{r}}}{m_{1}+m_{\mathrm{r}}}}\right)$. The accuracy of this strategy can be evaluated by calculating the value of $\left(r_{\mathrm{A}}\right)$ after the dynamic analysis of the collision problem using equation (2), and comparing it with the target one $\left(r_{\mathrm{T}}\right)$; obviously, the closer, the higher the accuracy. This very simple and widely used model has been compared with more complicated solutions and has proven to provide comparable or even better accuracy [28-30].

\section{PROPOSED FORMULATION FOR ESTIMATING DAMPING}

\subsection{General remarks}

The proposed formulation follows basically the strategy in [14], although releasing some of their assumptions, namely, considering the influence of the equivalent stiffness of each building and the gap size $(d)$ but still neglecting the influence of the input. Regarding this last consideration, the influence of the input severity is taken into consideration through the relative impact velocity. At first, the modal analysis of the colliding buildings during their impact is performed and the second mode damping ratio is determined in terms of the damping parameters of the Kelvin-Voigt model and of the colliding buildings, analogously to the right equation (3). Then, the uncoupled scalar modal equations of motion are solved; the ensuing closed-form solutions allow deriving a numerical algorithm providing a relation between the damping ratio of the second mode and the coefficient of restitution. This process corresponds to equation (4) in the previous formulation.

\subsection{Modal analysis of two buildings during impact}

This subsection presents an analytical study on the behavior of two colliding slabs during the duration of the impact (contact) between them; a deeper description can be found in [11]. As discussed in the previous subsection, this study follows the one in [14], although incorporating the gap and considering, in a simplified way, the influence of the stiffness of the buildings. Figure 2 describes the analyzed mechanical model. 


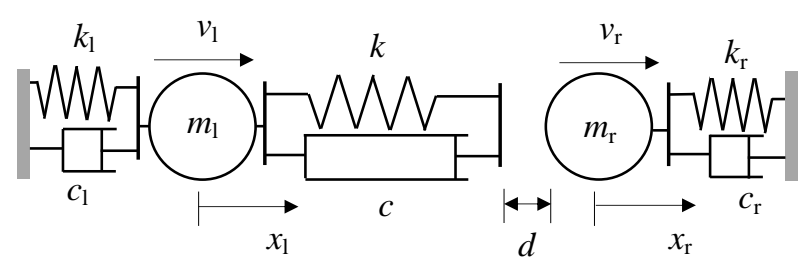

Figure 2. Considered mechanical model of the impact between two aligned slabs

Figure 2 employs the same notation than Figure 1; regarding the new symbols, $c_{1}$ and $c_{\mathrm{r}}$, and $k_{1}$ and $k_{\mathrm{r}}$ represent the equivalent damping, and stiffness of the left and right buildings, respectively. These equivalent values are not the actual parameters of the buildings, but those that better characterize, exclusively during the impact duration, the dynamic evolution of the colliding slabs. The corresponding equivalent undamped natural frequencies are given by $\omega_{\mathrm{l}}=\sqrt{\frac{k_{1}}{m_{\mathrm{l}}}}$ and $\omega_{\mathrm{r}}=\sqrt{\frac{k_{\mathrm{r}}}{m_{\mathrm{r}}}}$, respectively; analogously, the damping ratios are $\zeta_{\mathrm{l}}=\frac{c_{1}}{2 \sqrt{k_{\mathrm{l}} m_{\mathrm{l}}}}$ and $\zeta_{\mathrm{r}}=\frac{c_{\mathrm{r}}}{2 \sqrt{k_{\mathrm{r}} m_{\mathrm{r}}}}$. Noticeably, $\omega_{1}$ and $\omega_{\mathrm{r}}$ do not correspond to the fundamental frequencies of the colliding buildings, but merely to the equivalent frequencies of the colliding slabs during impact.

The ratio between the colliding masses is defined as $\mu=m_{1} / m_{\mathrm{r}}$; provided that both buildings belong to the same structural type, it can be reasonably assumed that the equivalent stiffness hold the same proportion than the mass: $\mu=m_{1} / m_{\mathrm{r}}=k_{\mathrm{l}} / k_{\mathrm{r}}$; therefore, $\omega_{\mathrm{l}}=\omega_{\mathrm{r}}$. As well, it can be rationally supposed that both buildings have the same damping ratio; then, it follows immediately that $\mu=c_{1} / c_{\mathrm{r}}$ (noticeably, these considerations are the same that lead to the simplified expressions for the fundamental period of a given building that are contained in the major design codes). Then, for convenience, dimensionless coefficients $\lambda$ and $\gamma$ are introduced: $\lambda=c_{\mathrm{r}} / c$ and $\gamma=k_{\mathrm{r}} / k$. Finally, the damped free motion of the 2-DOF system depicted at Figure 2 is governed by the linear equation $\mathbf{M} \ddot{\mathbf{x}}+\mathbf{C} \dot{\mathbf{x}}+$ $\mathbf{K} \mathbf{x}+\mathbf{b}=\mathbf{0}$, where the displacement vector $\mathbf{x}$, the constant vector $\mathbf{b}$, and the mass $(\mathbf{M})$, damping $(\mathbf{C})$ and stiffness (K) matrices are given by

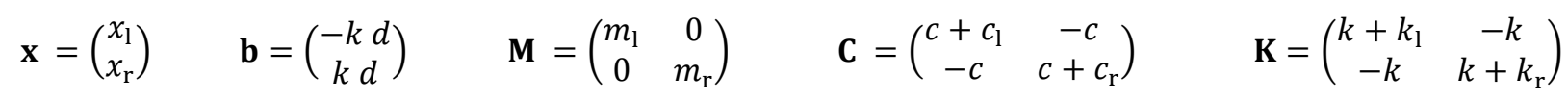

The modal analysis of this system provides the two undamped natural frequencies of the first and second modes:

$$
\omega_{1}=\omega_{1}=\omega_{\mathrm{r}} \quad \omega_{2}=\omega_{1} \sqrt{\frac{1+\mu+\mu \gamma}{\mu \gamma}}=\sqrt{k \frac{1+\mu+\mu \gamma}{\mu m_{r}}}
$$

The modal matrix is $\Phi=\left(\begin{array}{cc}1 & 1 \\ 1 & -\mu\end{array}\right)$. By using this matrix, the modal coordinates are introduced as $\mathbf{x}=\boldsymbol{\Phi} \eta$ : $x_{1}=\eta_{1}+\eta_{2}$ and $x_{\mathrm{r}}=\eta_{1}-\mu \eta_{2}$; the inverse relations are given by $\eta_{1}=\frac{1}{1+\mu}\left(\mu x_{1}+x_{\mathrm{r}}\right)$ and $\eta_{2}=\frac{1}{1+\mu}\left(x_{1}-x_{\mathrm{r}}\right)$. These equations show that the first mode corresponds to rigid-body motion, in the sense that does not generate any strain in the Kelvin-Voigt model $\left(\eta_{2}=0\right.$, implies that $\left.x_{1}=x_{\mathrm{r}}\right)$; this is consistent with the left equation (6). On the contrary, in the second mode both slabs move in opposite directions, thus causing strains in the Kelvin-Voigt model. Comparison between the left equation (3) and the right equation (6) highlights the similarity between this formulation and the previous one [14]; noticeably, if $\gamma=0, \omega_{2}$ is equal to the value $(\omega)$ from Anagnostopulos and if $\gamma \neq 0, \omega_{2}$ is higher.

After some algebra, the uncoupled scalar damped equations of motion in modal coordinates become:

$$
m_{1} \ddot{\eta}_{1}+c_{1} \dot{\eta}_{1}+k \mu \gamma \eta_{1}=0 \quad m_{\mathrm{r}} \mu \ddot{\eta}_{2}+\left[c(1+\mu)+c_{\mathrm{r}} \mu\right] \dot{\eta}_{2}+k(1+\mu+\mu \gamma) \eta_{2}=k d
$$

The first equation in (7) confirms that the first mode damping is only contributed by the buildings damping; this is coherent with the aforementioned absence of strains in the Kelvin-Voigt model. The damping ratios of the first and second modes are given by 


$$
\zeta_{1}=\zeta_{1}=\zeta_{\mathrm{r}} \quad \zeta_{2}=\zeta_{1} \frac{1+\mu+\mu \lambda}{\sqrt{(1+\mu+\mu \gamma) \mu}} \frac{\sqrt{\gamma}}{\lambda}=\zeta_{\mathrm{r}} \frac{1+\mu+\mu \lambda}{\sqrt{(1+\mu+\mu \gamma) \mu}} \frac{\sqrt{\gamma}}{\lambda}=\frac{c(1+\mu)+\mu c_{\mathrm{r}}}{2 \sqrt{k(1+\mu+\mu \gamma) \mu m_{\mathrm{r}}}}
$$

The last relation in equation (8) plays the same role as the second equation (3) in the study by Anagnastopulos; more precisely, if $\lambda=\gamma=0$, both expressions are alike.

\subsection{Closed-form solution of the equations of motion}

The closed-form solution for the system depicted in Figure 2 can be obtained by solving the scalar equations (7) and changing from modal (with subindexes 1 and 2) to physical (with subindexes $l$ and $r$ ) coordinates. At the onset of impact, the initial conditions are $x_{1}(0)=x_{0}, x_{\mathrm{r}}(0)=x_{0}-d, \dot{x}_{1}(0)=v_{\mathrm{l}}$ and $\dot{x}_{\mathrm{r}}(0)=v_{\mathrm{r}}$. In modal coordinates, these relations become $\eta_{1}(0)=x_{0}-\frac{d}{1+\mu}, \eta_{2}(0)=\frac{d}{1+\mu}, \dot{\eta}_{1}(0)=\frac{\mu v_{1}+v_{\mathrm{r}}}{1+\mu}$ and $\dot{\eta}_{2}(0)=\frac{v_{1}-v_{\mathrm{r}}}{1+\mu}$; noticeably, since $v_{1} \geq$ $v_{\mathrm{r}}$, then $\dot{\eta}_{2}(0) \geq 0$. The closed-form solutions of the equations (7) that fulfill these conditions are

$$
\begin{aligned}
& \eta_{1}(t)=e^{-\zeta_{1} \omega_{1} t}\left[\frac{\dot{\eta}_{1}(0)+\zeta_{1} \omega_{1} \eta_{1}(0)}{\omega_{1}\left(1-\zeta_{1}^{2}\right)^{1 / 2}} \sin \omega_{1}\left(1-\zeta_{1}^{2}\right)^{1 / 2} t+\eta_{1}(0) \cos \omega_{1}\left(1-\zeta_{1}^{2}\right)^{1 / 2} t\right] \\
& \eta_{2}(t)=e^{-\zeta_{2} \omega_{2} t}\left[\frac{\dot{\eta}_{2}(0)+\zeta_{2} \omega_{2} \eta_{2}(0)}{\omega_{2}\left(1-\zeta_{2}^{2}\right)^{1 / 2}} \sin \omega_{2}\left(1-\zeta_{2}^{2}\right)^{1 / 2} t+\eta_{2}(0) \cos \omega_{2}\left(1-\zeta_{2}^{2}\right)^{1 / 2} t\right]+\frac{k d}{\omega_{2}^{2} m_{1}}
\end{aligned}
$$

Then, the closed-form solutions in physical coordinates can be easily obtained after the relations $x_{1}=\eta_{1}+\eta_{2}$ and $x_{\mathrm{r}}=\eta_{1}-\mu \eta_{2}$.

\subsection{Impact duration}

The impact initiates when $x_{1}-x_{\mathrm{r}}=d(t=0)$ and finishes next time this condition is fulfilled again $\left(t=t_{\text {imp }}\right)$; the coefficient of restitution can be determined after equation (2), where the final and initial velocities correspond to instants $t=0$ and $t=t_{\text {imp }}$, respectively. Since $x_{1}-x_{\mathrm{r}}=(1+\mu) \eta_{2}$, this analysis can be conducted regardless of $\eta_{1}$; the conditions of $\eta_{2}$ for the start and end of impact become, respectively

$$
\eta_{2}(0)=\frac{d}{1+\mu} \quad \eta_{2}\left(t_{\text {imp }}\right)=\frac{d}{1+\mu}
$$

The right equation (10) can be solved for $t_{\text {imp }}$ by any standard iterative procedure, e.g. Newton-Raphson. Equations (7) and (9) show that, since $\eta_{2}(0)$ and $\dot{\eta}_{2}(0)$ are positive, equation (10) corresponds to a segment of a half wave of the free response of a damped SDOF system; therefore, the initial value of $t_{\text {imp }}$ in the aforementioned iterative procedure can be selected as $\frac{\pi}{\omega_{2}\left(1-\zeta_{2}^{2}\right)^{1 / 2}}$, given that such instant corresponds to the right end of that wave. Then, the time derivative can be taken from equation (13) (subsection 3.5). These considerations show that, although equation (10) has infinite solutions, the desired one can be obtained after only a few iterations.

When $c_{1}=c_{\mathrm{r}}=0$, the right equation (10) can be solved without iterating since, in that case, this relation has a closed-form solution for $\zeta_{2}=0$. Then, it is solved using a power series expansion: $t_{\mathrm{imp}}\left(\zeta_{2}\right)=t_{\mathrm{imp}}(0)+\frac{t_{\mathrm{imp}}^{\prime}(0)}{1 !} \zeta_{2}+$ $\frac{t_{\text {imp }}^{\prime \prime}(0)}{2 !} \zeta_{2}^{2}+O\left(\zeta_{2}^{3}\right)[11]$

In the particular case when $d=0$, the condition (10) becomes $\eta_{2}\left(t_{\mathrm{imp}}\right)=0$; this equation has a closed-form solution:

$$
t_{\text {imp }}=\pi \sqrt{\frac{\mu m_{\mathrm{r}}}{k\left(1-\zeta_{2}^{2}\right)(1+\mu+\mu \gamma)}}=\frac{\pi}{\omega_{2}\left(1-\zeta_{2}^{2}\right)^{1 / 2}}=t_{\max } \quad(\text { if } d=0)
$$

Comparison with the value from $[14]\left(\pi / \omega\left(1-\zeta^{2}\right)^{1 / 2}\right)$ shows that, when $d=0$, the impact duration according to the proposed formulation is shorter.

\subsection{Determination of the damping parameter}


The coefficient of restitution is obtained by replacing $v_{\mathrm{l}}=\dot{x}_{\mathrm{l}}(0), v_{\mathrm{r}}=\dot{x}_{\mathrm{r}}(0), v_{\mathrm{l}}^{\prime}=\dot{x}_{\mathrm{l}}\left(t_{\mathrm{imp}}\right)$ and $v_{\mathrm{r}}^{\prime}=\dot{x}_{\mathrm{r}}\left(t_{\mathrm{imp}}\right)$ in equation (2):

$$
r=\frac{v_{\mathrm{r}}^{\prime}-v_{1}^{\prime}}{v_{1}-v_{\mathrm{r}}}=\frac{\dot{x}_{\mathrm{r}}\left(t_{\mathrm{imp}}\right)-\dot{x}_{1}\left(t_{\mathrm{imp}}\right)}{\dot{x}_{\mathrm{l}}(0)-\dot{x}_{\mathrm{r}}(0)}=\frac{\dot{\eta}_{2}\left(t_{\mathrm{imp}}\right)}{-\dot{\eta}_{2}(0)}
$$

Velocities $\dot{\eta}_{2}\left(t_{\mathrm{imp}}\right)$ and $\dot{\eta}_{2}(0)$ are calculated after the time derivative of the second equation (9):

$$
\dot{\eta}_{2}(t)=e^{-\zeta_{2} \omega_{2} t}\left[-\frac{\zeta_{2} \dot{\eta}_{2}(0)+\eta_{2}(0) \omega_{2}}{\left(1-\zeta_{2}^{2}\right)^{1 / 2}} \sin \omega_{2}\left(1-\zeta_{2}^{2}\right)^{1 / 2} t+\dot{\eta}_{2}(0) \cos \omega_{2}\left(1-\zeta_{2}^{2}\right)^{1 / 2} t\right]
$$

Equation (12) provides $r$ in terms of $\zeta_{2}$. Given that the objective of the proposed approach is obtaining $\zeta_{2}$ after $r_{\mathrm{T}}$, the solution might be obtained iteratively by the Newton-Raphson method. In this iteration, the solution by Anagnostopoulos (equation (4)) [14] can be used for the initial estimation of $\zeta_{2}$. Regarding the derivatives, since the actual derivative of $\zeta_{2}$ with respect to $r$ is not available, the derivative of equation (4) can be used instead. Noticeably, given that the dependence of $r$ with respect to $\zeta_{2}$ is monotonic and descendent (e.g. negative derivative), this equation has a single solution and the Newton-Raphson method shall perform satisfactorily.

In the previous formulation, equation (4) shows that the relation between $r$ and $\zeta$ is independent on the stiffness of the model $(k)$ and on the masses of the colliding bodies $\left(m_{1}\right.$ and $\left.m_{\mathrm{r}}\right)$; in the proposed formulation, the relation between $r$ and $\zeta_{2}$ is dependent on the ratios between the damping and stiffness of the structure and the model ( $\lambda$ and $\gamma$ ) and on the ratio between the masses of the colliding bodies $(\mu)$.

In the particular case when $d=0$, the coefficient of restitution can be determined without any iteration; the obtained result is $r_{\mathrm{T}}=e^{-\zeta_{2} \frac{\pi}{\left(1-\zeta_{2}^{2}\right)^{1 / 2}}}$. Since this relation matches equation (4) (although replacing $\zeta_{2}$ with $\zeta_{\mathrm{A}}$ ), it can be concluded that the proposed algorithm coincides with the previous one when $d=0$. Noticeably, the aforementioned difference in the impact duration has no effect in the relation between $\zeta_{2}$ and $r_{\mathrm{T}}$.

\subsection{Proposed algorithm for selecting the damping parameter}

As stated previously, the proposed algorithm consists in stating initially $r_{\mathrm{T}}$ and then obtaining $\zeta_{2}$ and $c_{\mathrm{p}}$, similarly to equations (3) and (4) in the formulation by Anagnostopoulos [14]. Apart from $r_{\mathrm{T}}$, the required input variables are the structural parameters $m_{\mathrm{l}}, c_{\mathrm{l}}, k_{\mathrm{l}}, \mu, \gamma$, and the initial conditions $v_{\mathrm{l}}$ and $v_{\mathrm{r}}$; regarding $x_{0}$, since the stiffness of the buildings is assumed to be constant during the impact (linear behavior), it has no influence. The algorithm consists basically in the two nested iteration loops described in Figure 3. The iterations are performed with the aforementioned Newton-Raphson algorithms. Once $r_{\mathrm{T}}$ is stated, an initial value of $\zeta_{2}$ is set. Then $t_{\mathrm{imp}}$ is obtained by solving iteratively equation (10), where $\eta_{2}$ is given in equation (9); after $t_{\text {imp }}$, a new value of $r$ is determined from equation (12), where $\dot{\eta}_{2}(t)$ is provided by equation (13). Once convergence is reached, $c_{\mathrm{p}}$ can be determined after the last equation (8).

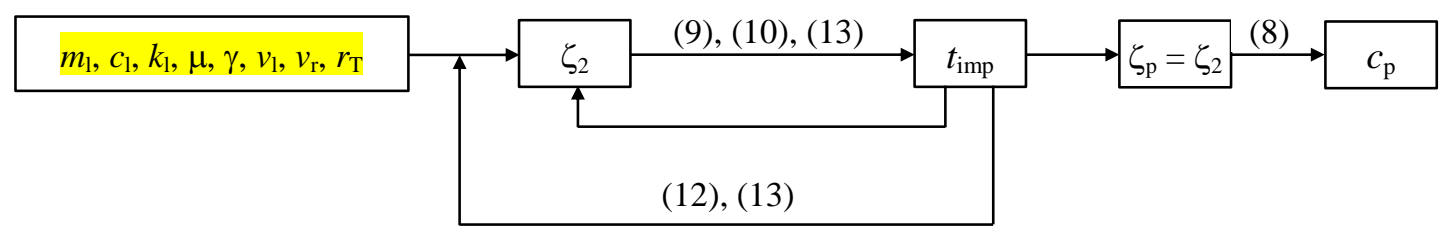

Figure 3. Flow chart of the proposed algorithm

Since the inner loop aims to solve the right equation (10), their tolerance is set as $\varepsilon_{\eta}=\varepsilon_{0}\left|\eta_{2}\left(t_{\text {max }}\right)-\frac{d}{1+\mu}\right|$, where $t_{\max }$ is defined in equation (11) and $\varepsilon_{0}$ is a dimensionless tolerance ratio to be chosen significantly smaller than 1. Regarding the outer loop, the iterations are performed with respect to the coefficient of restitution; given that is dimensionless and ranges between 0 and 1 , the tolerance $\left(\varepsilon_{\mathrm{r}}\right)$ shall be also considerably lower than 1 .

\section{COMPARISON WITH THE PREVIOUS FORMULATION}




\subsection{Description of the comparing parametric study}

This section presents a simplified parametric study on the collision between two single-story single-bay 2-D RC frames connected with a Kelvin-Voigt model. The main objective of this study is to assess the influence of the input parameters of the proposed algorithm (Figure 3), and thus to investigate its efficiency, mainly compared with the previous formulation.

Figure 4.a represents the colliding frames. No seismic excitation is considered, the collision is generated by separating initially each frame and then releasing them, as shown by Figure 4.b. Distances $a_{1}$ and $a_{\mathrm{r}}$ represent such initial outward displacements. Noticeably, in the general formulation described in section 3 , any set of values of $x_{0}$, $v_{1}$ and $v_{\mathrm{r}}$ can actually occur; conversely, in the cases analyzed in this section, the absence of excitation and the assumption that the velocities of both frames are simultaneously zero (Figure 4.b), makes that $x_{0}$ can be calculated after $a_{1}$ and $a_{\mathrm{r}}$. In other words, not any collection of values of $x_{0}, a_{1}$ and $a_{\mathrm{r}}$ is viable. It is considered that this limitation does not reduce significantly the scope of this study.

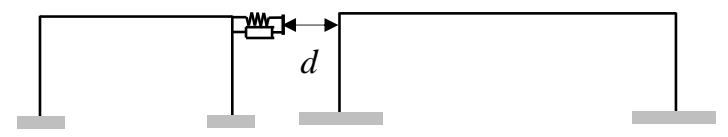

(a) Analyzed frames

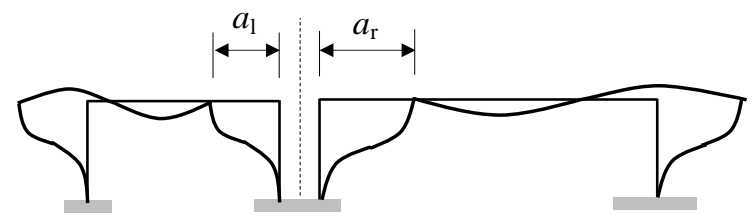

(b) Initial situation

Figure 4. Colliding frames considered in the comparison parametric study

The Kelvin-Voigt model damping is chosen according to both the previous and proposed formulations. The behavior of the frames is assumed to be linear, and, by using ordinary static and kinematic condensation, such frames are modeled as SDOF systems. Under these assumptions, the free (unforced) equations of motion for both contact and separation conditions have closed-form solutions.

The parameters of the study are: gap size $d$, initial separations $a_{1}$ and $a_{\mathrm{r}}$, damping ratios $\zeta_{\mathrm{l}}$ and $\zeta_{\mathrm{r}}\left(\zeta_{\mathrm{l}}=\zeta_{\mathrm{r}}\right)$, mass ratio $\mu$, stiffness ratio $\gamma$, and target coefficient of restitution $r_{\mathrm{T}}$. The comparison between the previous and the proposed approaches is established in terms of the obtained coefficients of restitution $r_{\mathrm{A}}$ and $r_{\mathrm{p}}$, respectively. Such coefficients are determined after the dynamic analyses (equation (2)) by using the values of $\zeta$ provided by the Anagnostopoulos $\left(\zeta_{\mathrm{A}}\right)$ and the proposed formulations $\left(\zeta_{\mathrm{p}}\right)$, respectively; the closer to the target value, the better the performance of the corresponding formulation.

\subsection{Colliding frames considered in the parametric study}

The geometrical and mechanical parameters of the left frame are described next; those of the right one are determined after the ratio $\mu$. The height and span-length are 3.2 and $5 \mathrm{~m}$, respectively. The beam section is $40 \times 50$ $\mathrm{cm}^{2}$ and the slabs are $15 \mathrm{~cm}$ deep; since each frame is assumed to be a part of a building RC structure, the beams are modeled as T-section members with effective width $140 \mathrm{~cm}$ [31]. All the columns are $60 \times 60 \mathrm{~cm}^{2}$; they are assumed to be clamped to the foundation. After this information, it follows that the mass and stiffness parameters of the left frame are $m_{1}=25136 \mathrm{~kg}$ and $k_{1}=87.96 \mathrm{kN} / \mathrm{mm}$. The moduli of deformation of concrete and steel are $25.743 \mathrm{GPa}$ and $200 \mathrm{GPa}$, respectively; the concrete cracking is taken into consideration by reducing the moments of inertia of beams and columns by factors 0.5 and 0.7 , respectively [32]. Then, the undamped natural frequency of each frame is $\omega_{\mathrm{l}}=\omega_{\mathrm{r}}=\sqrt{\frac{k_{\mathrm{r}}}{m_{\mathrm{r}}}}=\sqrt{\frac{87960 \times 1000}{25136}}=59.15 \frac{\mathrm{rad}}{\mathrm{s}}$; thus their natural period, is $2 \pi / 59.15=0.1062 \mathrm{~s}$. Noticeably, this value confirms the practical feasibility of the chosen parameter values.

\subsection{Selection of the parameters values}

The stiffness parameter of the Kelvin-Voigt model $(k)$ is selected, according to the aforementioned previous studies, referred to the axial stiffness $E A / L$ of the left colliding slab; $E, A$ and $L$ represent their modulus of deformation, cross section and length, respectively. Three values of $k$ are considered: $10 E A / L, E A / L$ and $0.1 E$ $A / L[12,13]$. In this frame, $E A / L=2111 \mathrm{kN} / \mathrm{mm}$; hence, the corresponding three values of $k$ are 21109,2111 and $211 \mathrm{kN} / \mathrm{mm}$. They correspond to $\gamma=0.00417,0.0417$ and 0.417 , respectively. Three values of gap are taken: $d$ 
$=10 / 20 / 30 \mathrm{~mm}$. Two levels of damping of the frames are selected: $\zeta_{1}=\zeta_{\mathrm{r}}=0 / 0.05$. Regarding $\mu$, both symmetric $(\mu=1)$ and asymmetric $(\mu=2)$ situations are contemplated. Finally, three initial displacements are considered: $a_{1}=$ $a_{\mathrm{r}}=20 / 30 / 40 \mathrm{~mm}$. These values intend to represent most of the situations actually occurring in real cases; it is not believed necessary to select different values for $a_{1}$ and $a_{\mathrm{r}}$, since the differences of masses (if $\mu=2$ ) sufficiently represent unbalanced impacts (i.e. with different momenta). By combining these values of $\mu, \gamma, d$ and $a_{1}\left(a_{1}=a_{\mathrm{r}}\right)$, 108 cases are analyzed. Regarding the coefficient of restitution, the full range $(0-1)$ is considered. As for the nested iteration loops (Figure 3), the tolerances for the inner and outer loops are $\varepsilon_{0}=0.01$ and $\varepsilon_{\mathrm{r}}=0.001$, respectively.

\subsection{Numerical results of the parametric study}

This subsection summarizes the results of the analyses that have been described in the previous subsection. Figure 5 displays an example of the left frame displacement history. This plot corresponds to initial displacement $40 \mathrm{~mm}$, gap size $10 \mathrm{~mm}$, and target coefficient of restitution 0.7 . The horizontal dashed line corresponds to the threshold for contact $\left(x_{1}=d / 2\right)$; the values of $x_{1}$ above this bound correspond to impact.

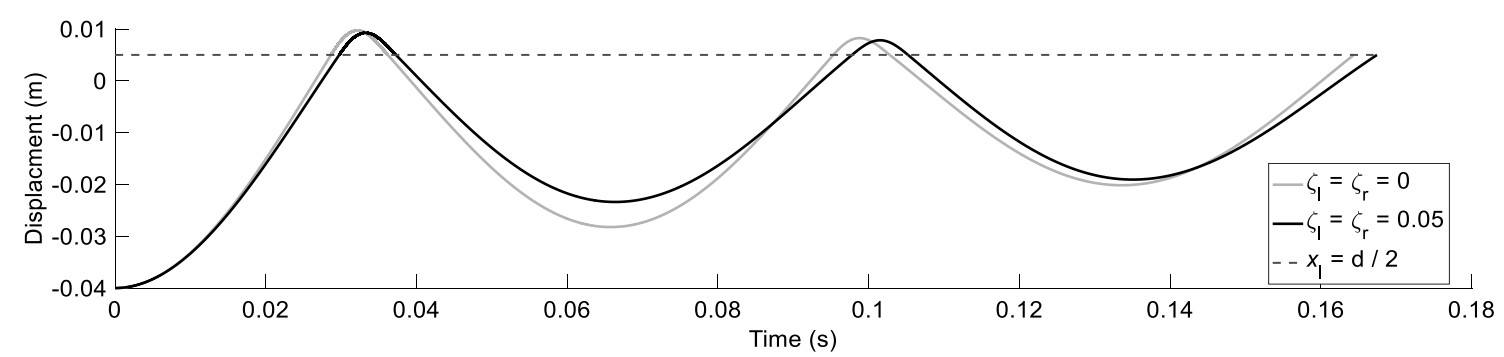

Figure 5. Time history of $x_{1}$ for $a_{1}=a_{\mathrm{r}}=40 \mathrm{~mm}, d=10 \mathrm{~mm}, \mu=1, \gamma=0.0417$, and $r_{\mathrm{T}}=0.7$

Figure 5 shows that, in the consecutive impacts, the maximum penetration decrease significantly; as well, each free vibration segment (below the dashed line) reveals the reduction caused by the energy absorbed in the previous impact. This is coherent with the physical behavior of the system.

The results of the simplified parametric study are presented in terms of plots of ratios $r_{\mathrm{p}} / r_{\mathrm{T}}$ and $r_{\mathrm{A}} / r_{\mathrm{T}}$ vs. $r_{\mathrm{T}}$; the closer to 1, the better the performance of the corresponding formulation. Figure 6 displays representative results; the reference [11] contains a wider collection of plots. The values of $r_{\mathrm{p}}$ and $r_{\mathrm{A}}$ are determined accounting only for the first impact, being the strongest one.

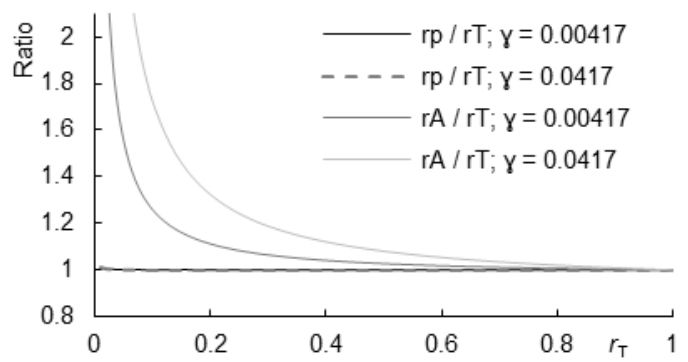

(a) $d=30 \mathrm{~mm}, a_{1}=a_{\mathrm{r}}=20 \mathrm{~mm}$

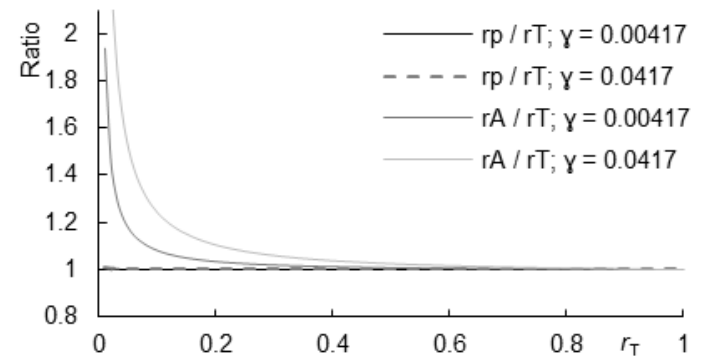

(b) $d=10 \mathrm{~mm}, a_{1}=a_{\mathrm{r}}=40 \mathrm{~mm}$

Figure 6. Ratios $r_{\mathrm{p}} / r_{\mathrm{T}}$ and $r_{\mathrm{A}} / r_{\mathrm{T}}$ between the proposed and the previous formulations, for $\zeta_{\mathrm{l}}=\zeta_{\mathrm{r}}=$ 0.05 and $\mu=1$

\subsection{Concluding remarks}

Figure 6 and [11] show that, $r_{\mathrm{p}} \approx r_{\mathrm{T}}$; in other words, in the conducted parametric study, the proposed formulation is virtually exact. This circumstance is expected, because, given that the behavior of the frames is linear and there is no continuous excitation, in this case the proposed approach does not contain any simplification other than the approximate determination of $t_{\text {imp. }}$. It is also apparent that the former formulation [14] exhibits rather good performance, except for high values of $\gamma$ and small values of $r_{\mathrm{T}}$. The separate influences of the parameters of the study are discussed next. 
- Target coefficient of restitution $\left(\boldsymbol{r}_{\mathrm{T}}\right)$. The plots exhibit two clear trends: (i) the previous formulation provides values of $r$ that are greater than foreseen $\left(r_{\mathrm{A}} \geq r_{\mathrm{T}}\right)$, and (ii) when $r_{\mathrm{T}}$ approaches $0 / 1$ the ratio $r_{\mathrm{A}} / r_{\mathrm{T}}$ comes close to $0 / 1$. The first trend means that [14] overestimates the coefficient of restitution and, hence, underestimates the actual damping during the collision. In other words, the former approach would predict that damage is more spread in the full body of the colliding buildings; conversely, the proposed algorithm envisages that the damage will be more concentrated in the vicinities of the impact areas. Finally, the second trend indicates that the proposed approach takes more advantage (compared to the previous one) in plastic impacts.

- Ratio between the stiffness of frame and of Kelvin-Voigt model $(\gamma)$. All the plots show that, the higher $\gamma$, the bigger the difference. The explanation for this trend is obvious, given that the previous study assumed $\gamma=0$ [14]. The work [22] had announced that the influence of the stiffness of the main structure could be relevant; this statement is corroborated in this research.

- Gap size $(\boldsymbol{d})$. In all the plots, the difference increases with $d$. This is coherent with the previous observation that the proposed formulation and the previous one [14] are coincident for $d=0$.

- Initial separation $\left(\boldsymbol{a}_{\mathbf{l}}=\boldsymbol{a}_{\mathrm{r}}\right)$. In all the cases, the difference decreases as the initial separation increases. This can be read as less discrepancy between both formulations for stronger collisions. This is logical, since, for more violent impacts, the neglected parameters play comparatively less important roles. Noticeably, these remarks refer indirectly to the relative velocity at the onset of pounding $\left(v_{1}-v_{\mathrm{r}}\right)$, given that larger initial separation leads to higher impact velocity.

- Ratio between the left and right frame mass $(\mu)$. The plots show rather little sensitivity to $\mu$. This is consistent, since this parameter has been accounted for in similar ways in both approaches.

- Frame damping $\left(\zeta_{1}=\zeta_{\mathrm{r}}\right)$. All the plots show that, the higher the frame damping, the bigger the difference. The justification for this tendency is apparent, since in the previous study [14] the frame damping was not taken into consideration.

\section{CRITERIA FOR SELECTING $v_{\mathrm{l}}$ AND $v_{\mathrm{r}}$}

As described in Figure 3, the input parameters for the proposed algorithm can be classified into those dealing with the structural properties (mass $m_{\mathrm{l}}$, damping $c_{\mathrm{l}}$, stiffness $k_{\mathrm{l}}$, ratios $\mu$ and $\gamma$, and target coefficient of restitution $r_{\mathrm{T}}$ ) and those referring to the conditions at the onset of collision (impact velocities $v_{1}$ and $v_{\mathrm{r}}$ ). The parameters in the first group can be chosen directly; conversely, the selection of $v_{1}$ and $v_{\mathrm{r}}$ is less obvious, given that such velocities are not known in advance, like the input severity. Therefore, this section offers criteria for choosing $v_{1}$ and $v_{\mathrm{r}}$ by analyzing their influence on $c_{\mathrm{p}}$. The obtained results are summarized in Figure 7 and Figure 8; Figure 7 analyzes the case when $v_{1}=-v_{\mathrm{r}}$ and Figure 8 studies the influence of $v_{1}+v_{\mathrm{r}}$. Provided that $\mu=1$, the first case corresponds to symmetric impact (i.e. opposite momenta, $m_{1} v_{1}=-m_{\mathrm{r}} v_{\mathrm{r}}$ ), and the second case represents asymmetric impact. Figure 7 and Figure 8 display plots of $c_{\mathrm{p}}$ vs. $v_{1}$ and $v_{1}+v_{\mathrm{r}}$, respectively. The influence of $\mu, \zeta, \gamma$ and $d$ is analyzed; regarding $m_{1}$ and $k_{1}$, are not specifically addressed because their influence is rather obvious since, by assuming constant $\mu$, they behave basically as scale factors. 


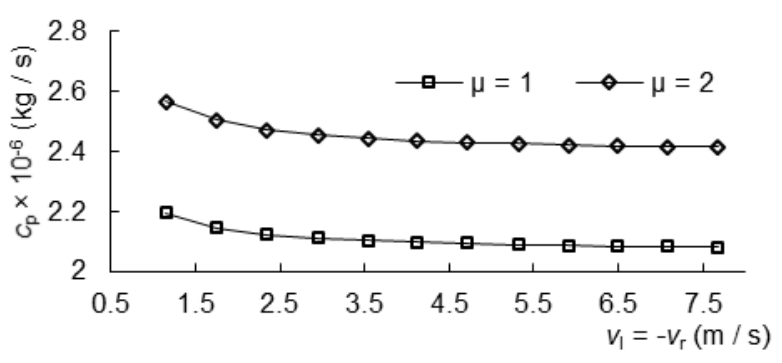

(a) $d=10 \mathrm{~mm}, \zeta_{\mathrm{l}}=\zeta_{\mathrm{r}}=0, \gamma=0.042$

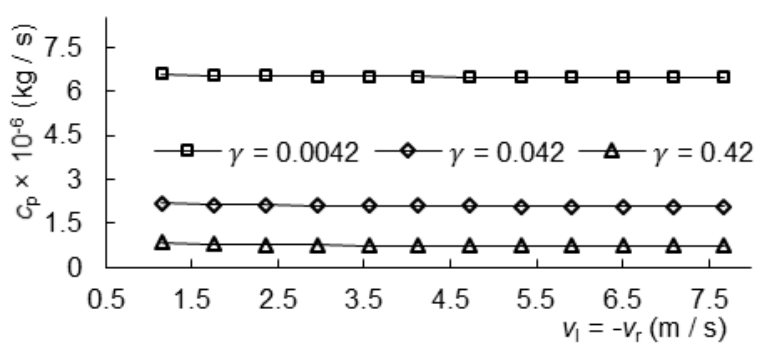

(c) $d=10 \mathrm{~mm}, \mu=1, \zeta_{\mathrm{l}}=\zeta_{\mathrm{r}}=0$

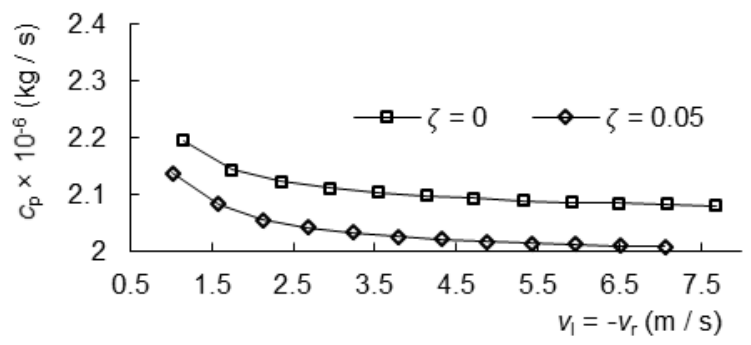

(b) $d=10 \mathrm{~mm}, \mu=1, \gamma=0.042$

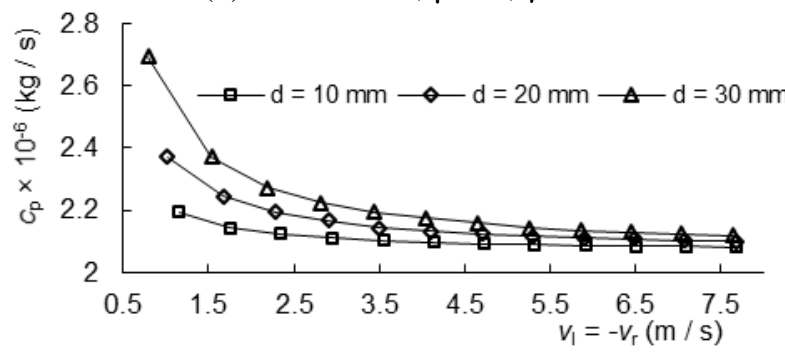

(d) $\mu=1, \zeta_{\mathrm{l}}=\zeta_{\mathrm{r}}=0, \gamma=0.042$

Figure 7. Kelvin-Voigt model damping variation with respect to $v_{1}\left(v_{1}+v_{\mathrm{r}}=0\right)$ according to the proposed formulation $\left(r_{\mathrm{T}}=\right.$ $0.53)$

Figure 7 shows that $c_{\mathrm{p}}$ is little sensitive to $v_{\mathrm{l}}$, in the sense that tends to stabilize when $v_{1}$ grows. Figure $7 . \mathrm{d}$ points out that this stabilization depends mainly on $d$; higher values of $d$ generate latter stabilization (i.e. for greater velocity). This trend corroborates the observations after Figure 6, in terms of the influence of the initial separation. Therefore, it is recommended to select $v_{1}$ in between 1.5 and $10.5 \mathrm{~m} / \mathrm{s}$; inside this range, smaller values are suggested if $d$ is small and no violent impact is expected, and greater values are advised in the opposite situation.

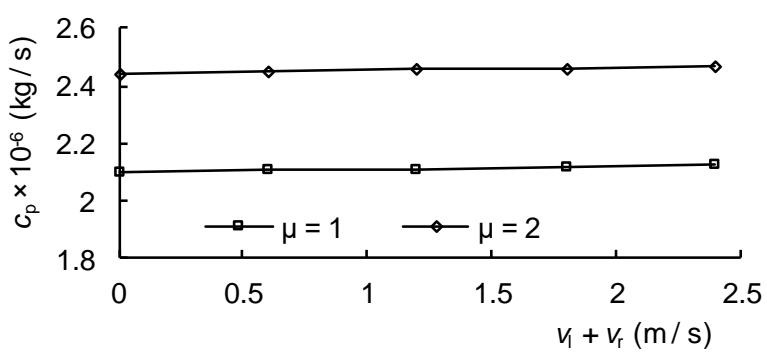

(a) $d=10 \mathrm{~mm}, \zeta_{\mathrm{l}}=\zeta_{\mathrm{r}}=0, \gamma=0.042$

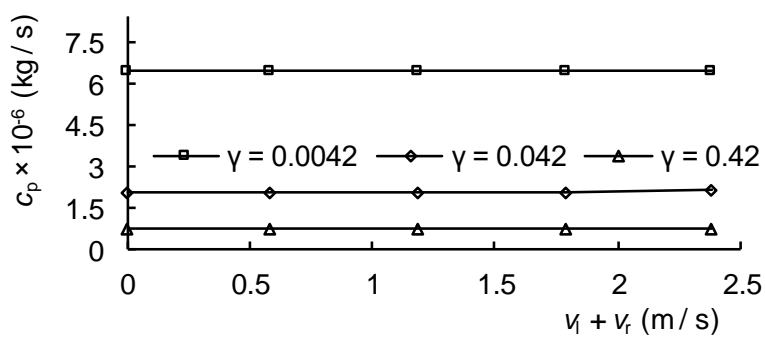

(c) $d=10 \mathrm{~mm}, \mu=1, \zeta_{\mathrm{l}}=\zeta_{\mathrm{r}}=0$

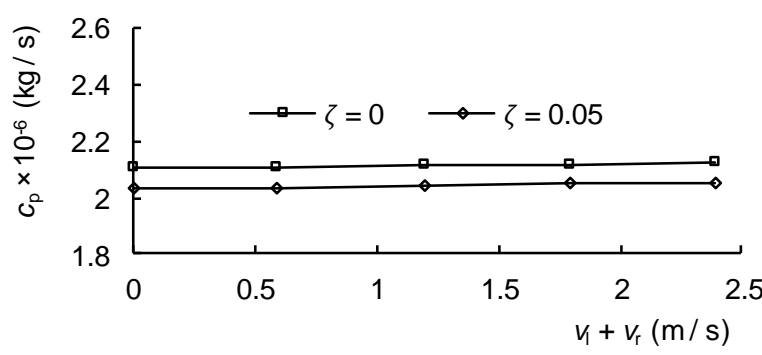

(b) $d=10 \mathrm{~mm}, \mu=1, \gamma=0.042$

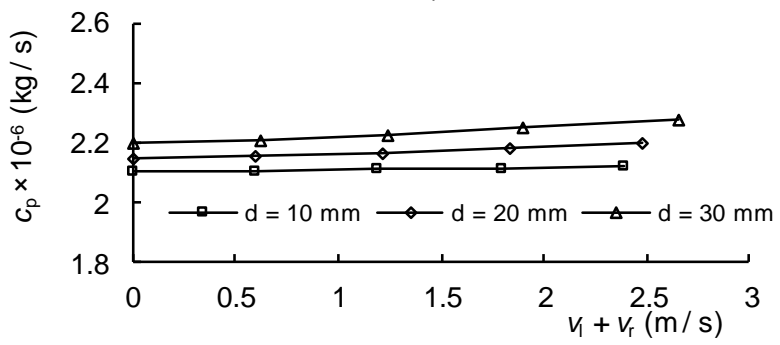

(d) $\mu=1, \zeta_{\mathrm{l}}=\zeta_{\mathrm{r}}=0, \gamma=0.042$

Figure 8. Kelvin-Voigt model damping variation with respect to $v_{1}+v_{\mathrm{r}}$ according to the proposed formulation $\left(r_{\mathrm{T}}=0.53\right)$

Plots from Figure 8 are basically flat. This shows that the influence of $v_{1}+v_{\mathrm{r}}$ can be neglected; in other words, it can be assumed that $v_{1}+v_{\mathrm{r}}=0$.

This section shows that the nested iteration loops described in Figure 3 need to be carried out only one time for each impact analysis; in other words, this operation can be performed during the pre-process stage. The reason is that, although the proposed algorithm requires the estimation of the relative impact velocities, Figure 7 and Figure 8 show that their sensitivity to these parameters is low and can be neglected. Therefore, the upgrade provided by the proposed algorithm is obtained at a small computational cost. 


\section{NUMERICAL EXAMPLES ON TWO MULTI-STORY MULTI-BAY FRAMES}

\subsection{Colliding frames}

This section presents two examples on the impact between two pairs of 2-D RC frames undergoing seismic excitation. These examples are aimed to highlight the capacity of the proposed formulation to reproduce adequately the impact effect, in the sense of obtaining a coefficient of restitution close to its target value.

The dynamic behavior of the frames is assumed to be linear and is described with the SeismoStruct code [33]. Figure 9 depicts the main characteristics of the colliding frames.

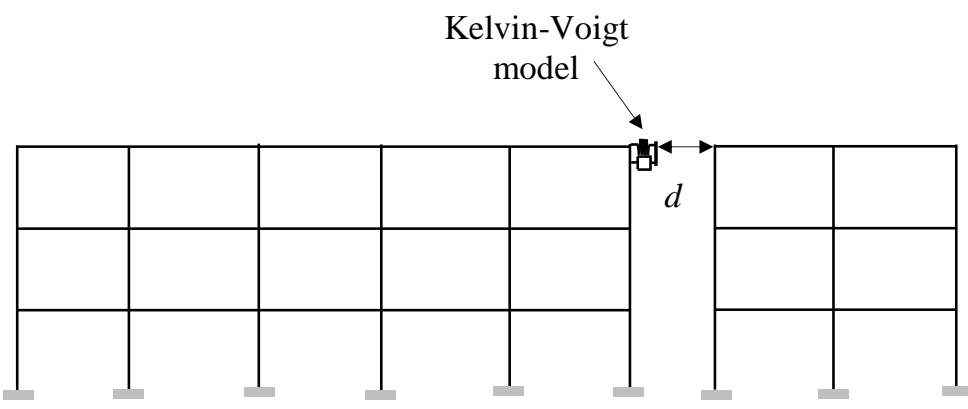

(a) 3-story 5-bay and 3-story 2-bay frames

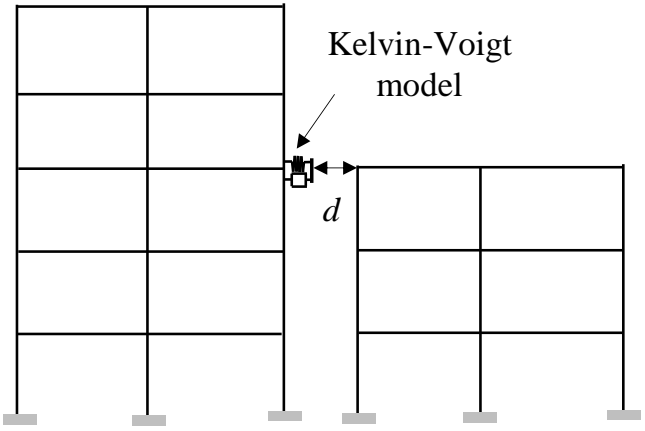

(b) 5-story 2-bay and 3-story 2-bay frames

Figure 9. Pairs of colliding frames

Figure 9 shows that in the first pair of frames, the left one has three stories and five bays and the right one has three stories and two bays; in the second pair, both frames have two bays and the left and right ones have five and three stories, respectively. The impact is described with single Kelvin-Voigt models located at the top colliding slabs; no impacts at other levels have been detected.

In all the cases, the span-length is $5 \mathrm{~m}$ and the story height is $3.2 \mathrm{~m}$. Each frame is assumed to be a part of a RC building that is composed of columns, slabs, and beams in two directions; the separation among the frames is $5 \mathrm{~m}$. These buildings have been designed for a high seismicity region [11]. The beams section is $40 \mathrm{~cm} \times 50 \mathrm{~cm}$ and the slabs are $15 \mathrm{~cm}$ deep; the beams are modeled as T-section members with effective width $105 \mathrm{~cm}$ [31]. Columns are assumed to be clamped to the foundation and have rectangular cross-section; Table 1 displays the corresponding transverse dimensions.

\begin{tabular}{|c|c|c|c|c|c|}
\hline \multicolumn{6}{|c|}{ Table 1. Cross section of the columns of the analyzed frames in the example simulation } \\
\hline Building & $\begin{array}{c}\mathbf{1}^{\text {st }} \text { floor } \\
\text { columns (cm) }\end{array}$ & $\begin{array}{c}\mathbf{2}^{\text {nd }} \text { floor } \\
\text { columns (cm) }\end{array}$ & $\begin{array}{c}\mathbf{3}^{\text {rd }} \text { floor } \\
\text { columns (cm) }\end{array}$ & $\begin{array}{c}\mathbf{4}^{\text {th }} \text { floor } \\
\text { columns (cm) }\end{array}$ & $\begin{array}{c}\mathbf{5}^{\text {th }} \text { floor } \\
\text { columns (cm) }\end{array}$ \\
\hline 3-story 5-bay & $60 \times 60$ & $55 \times 55$ & $50 \times 50$ & - & - \\
\hline 3-story 2-bay & $60 \times 60$ & $55 \times 55$ & $50 \times 50$ & - & - \\
\hline 5-story 2-bay & $60 \times 60$ & $55 \times 55$ & $50 \times 50$ & $45 \times 45$ & $40 \times 40$ \\
\hline
\end{tabular}

The characteristic value of concrete compressive strength is $f_{\mathrm{ck}}=30 \mathrm{MPa}$ and the modulus of deformation is 25.74 $\mathrm{GPa}$. The seismic weight of each frame corresponds to the load combination $D+0.2 L$, where $D$ and $L$ account for dead and live load, respectively; a deeper description can be found in [11]. Table 2 displays the natural periods and the modal mass ratios of the analyzed frames; noticeably, these quantities would also correspond to the buildings the frames belong to. The stiffness of columns and beams refer to gross sectional parameters reduced due to concrete cracking [32]. Data from Table 2 show a regular and expected behavior; comparison among the fundamental periods of the two 3-story buildings confirms that they are little sensitive to the number of bays. 


\begin{tabular}{|c|c|c|c|c|c|c|}
\hline \multicolumn{7}{|c|}{ Table 2. Natural periods (s) and modal mass ratios of the individual buildings in the example simulation } \\
\hline \multirow{3}{*}{ Mode No. } & \multicolumn{7}{|c|}{ Frame } \\
\cline { 2 - 7 } & 3-story 5-bay & 3-story 2-bay & 5-story 2-bay \\
\cline { 2 - 7 } & $\boldsymbol{T}_{\mathbf{i}}$ & $\boldsymbol{m}_{\mathbf{i}}{ }^{*} / \boldsymbol{m}$ & $\boldsymbol{T}_{\mathbf{i}}$ & $\boldsymbol{m}_{\mathrm{i}}$ / $\boldsymbol{m}$ & $\boldsymbol{T}_{\mathbf{i}}$ & $\boldsymbol{m}_{\mathrm{i}}{ }^{*} / \boldsymbol{m}$ \\
\hline $\mathbf{1}$ & 0.3744 & 0.8277 & 0.3587 & 0.8222 & 0.6415 & 0.7664 \\
\hline $\mathbf{2}$ & 0.1283 & 0.1207 & 0.1201 & 0.1243 & 0.2349 & 0.1262 \\
\hline $\mathbf{3}$ & 0.0750 & 0.0516 & 0.069 & 0.0535 & 0.1398 & 0.0490 \\
\hline $\mathbf{4}$ & - & - & - & - & 0.0917 & 0.0479 \\
\hline $\mathbf{5}$ & - & - & - & - & 0.0794 & 0.0000 \\
\hline
\end{tabular}

\subsection{Seismic inputs}

Two ground motions are considered. Both accelerograms correspond to the Northridge earthquake (January 17, 1994); the first one was registered in Saticoy St. station, component USC90003, and the second one belongs to W Pico Canyon station, component USC90056. In these inputs, the PGA is $0.48 \mathrm{~g}$ and $0.455 \mathrm{~g}$, respectively. Deeper considerations can be found in [11]. Figure 10 displays both accelerograms. Figure 10 shows that the left accelerogram is not pulse-like, but the right one actually is [11]. These choices aim to obtain sound conclusions, since the presence of velocity pulses is relevant.

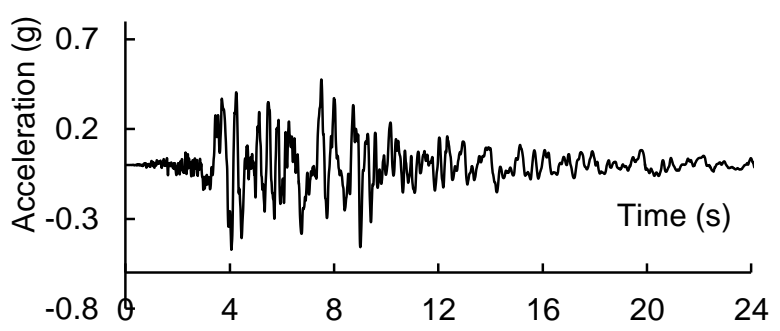

(a) Northridge Saticoy input for 3-story 5-bay and 3story 2-bay frames

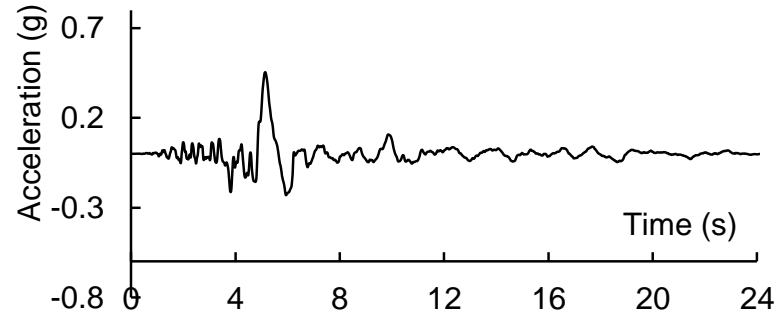

(b) Northridge W Pico Canyon input for 5-story 2-bay and 3-story 2-bay frames

Figure 10. Time histories of the seismic inputs

\subsection{Application of the proposed formulation}

For the implementation of the proposed algorithm, the equivalent mass $\left(m_{1}\right.$ and $\left.m_{\mathrm{r}}\right)$ and the stiffness $\left(k_{1}\right.$ and $\left.k_{\mathrm{r}}\right)$ of the colliding slabs are to be obtained. The mass is determined as in ordinary lumped-masses models; regarding the stiffness, the situation is less obvious, because the proposed formulation represents the colliding structures as SDOF systems (Figure 2). To clarify this issue, Figure 11 displays time zoom views of floor displacement time histories during the strongest collision; Figure 11.a and Figure 11.b refer to the left 3-story 5-bay and 5-story 2-bay frames, respectively (Figure 9). Figure 11 shows that the dynamic behavior during the impact duration follows basically the first mode, being little affected by the local effect of the impact force on the colliding level. Therefore, the equivalent stiffness of each frame is obtained as the ratio between a single static horizontal force acting on the impact level and its lateral displacement. This consideration shows that, although the estimation of $k_{1}$ and $k_{\mathrm{r}}$ requires carrying out static analyses, such analyses are simple and can be performed with the structural model of the colliding buildings that must be built anyway. 


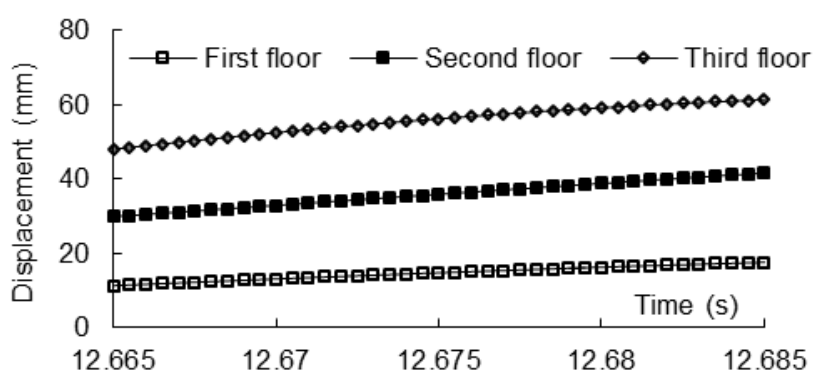

(a) 3-story 5-bay frame under Northridge Saticoy input

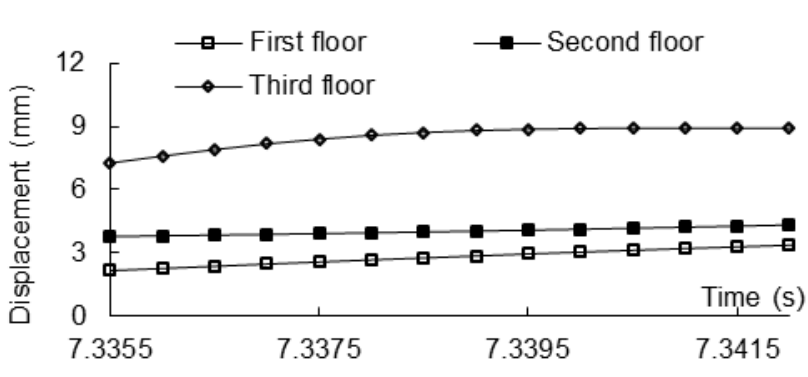

(b) 5-story 2-bay frame under Northridge W Pico Canyon input

Figure 11. Time histories of relative displacements of frame stories during the impact

In the 3-story 5-bay frame, the mass of the third floor slab is $117598 \mathrm{~kg}$ and the stiffness is $49.47 \mathrm{kN} / \mathrm{mm}$, in the 3story 2-bay frame such quantities are $47632 \mathrm{~kg}$ and $21.86 \mathrm{kN} / \mathrm{mm}$, and in the 5-story 2-bay frame the slab mass is $50029 \mathrm{~kg}$ and the equivalent stiffness is $22.00 \mathrm{kN} / \mathrm{mm}$. In all the cases, $d=20 \mathrm{~mm}$. In the case shown in Figure 9.a, the ratio $\mu$ is determined by dividing the left and right mass and stiffness; the obtained results are 2.469 and 2.263 , respectively. Given that both results are close, the aforementioned assumption $\left(m_{1} / m_{\mathrm{r}}=k_{1} / k_{\mathrm{r}}\right)$ holds approximately; hence, the average value of $\mu$ is considered in the analysis: $\mu=2.366$. In the case shown in Figure 9.b, the situation is similar; the corresponding values of $\mu$ are 1.05 and 1.00 , respectively and the average value that is adopted in the analysis is $\mu=1.03$. Regarding the frames damping, two options are contemplated: no damping $\left(\zeta_{1}=\zeta_{\mathrm{r}}=0\right)$ and 5\% of critical $\left(\zeta_{1}=\zeta_{\mathrm{r}}=0.05\right)$. Concerning the impact velocities, $v_{1}+v_{\mathrm{r}}=0$ is assumed, as suggested in section 5; then $v_{1}$ is selected iteratively by aiming to obtain a satisfactory agreement between $r_{\mathrm{T}}$ and the coefficient of restitution calculated from the results of the dynamic analyses. The selected values of such velocities are $2 \mathrm{~m} / \mathrm{s}$ (Figure 9.a, $\zeta_{1}=\zeta_{\mathrm{r}}=0$ ), $9 \mathrm{~m} / \mathrm{s}$ (Figure 9.a, $\zeta_{1}=\zeta_{\mathrm{r}}=0.05$ ), $10 \mathrm{~m} / \mathrm{s}$ (Figure 9.b, $\zeta_{1}=\zeta_{\mathrm{r}}=0$ ), and 9 $\mathrm{m} / \mathrm{s}$ (Figure 9.b, $\left.\zeta_{1}=\zeta_{\mathrm{r}}=0.05\right)$. For each of these four cases, the stiffness of the Kelvin-Voigt model is taken as $k=$ 211.1, 2111, 6558 and $6558 \mathrm{kN} / \mathrm{mm}$, respectively. Like in section 4, the tolerances for the inner and outer loops are $\varepsilon_{0}=0.01$ and $\varepsilon_{\mathrm{r}}=0.001$, respectively. The target value of the coefficient of restitution is $r_{\mathrm{T}}=0.53$. To sum up, Table 3 displays the parameters of the proposed algorithm.

\begin{tabular}{|c|c|c|c|c|c|c|c|c|c|}
\hline \multicolumn{1}{|c|}{ Table 3. Input parameters of the proposed algorithm in the example simulation } \\
\hline Case & $\boldsymbol{m}_{\mathbf{l}}(\mathbf{k g})$ & $\boldsymbol{c}_{\mathbf{l}}(\mathbf{k g} / \mathbf{s})$ & $\boldsymbol{k}_{\mathbf{l}}(\mathbf{k N} / \mathbf{m m})$ & $\boldsymbol{\mu}$ & $\boldsymbol{\gamma}$ & $\boldsymbol{d}(\mathbf{m m})$ & $\boldsymbol{v}_{\mathbf{1}}(\mathbf{m} / \mathbf{s})$ & $\boldsymbol{v}_{\mathbf{r}}(\mathbf{m} / \mathbf{s})$ & $\boldsymbol{r}_{\mathrm{T}}$ \\
\hline Figure 9.a, $\zeta_{\mathrm{l}}=\zeta_{\mathrm{r}}=0$ & 117598 & 0 & 49.47 & 2.366 & 0.1036 & 20 & 2 & -2 & 0.53 \\
\hline Figure 9.a, $\zeta_{\mathrm{l}}=\zeta_{\mathrm{r}}=0.05$ & 117598 & $1.535 \times 10^{5}$ & 49.47 & 2.366 & 0.0104 & 20 & 9 & -9 & 0.53 \\
\hline Figure 9.b, $\zeta_{\mathrm{l}}=\zeta_{\mathrm{r}}=0$ & 50029 & 0 & 22.00 & 1.030 & 0.0033 & 20 & 10 & -10 & 0.53 \\
\hline Figure 9.b, $\zeta_{\mathrm{l}}=\zeta_{\mathrm{r}}=0.05$ & 50029 & $1.020 \times 10^{5}$ & 22.00 & 1.030 & 0.0033 & 20 & 9 & -9 & 0.53 \\
\hline
\end{tabular}

The proposed algorithm (Figure 3) provides, for Figure 9.a, $c_{\mathrm{p}}=1.157 \times 10^{6} \mathrm{~kg} / \mathrm{s}$ and $c_{\mathrm{p}}=3.281 \times 10^{6} \mathrm{~kg} / \mathrm{s}$ for $\zeta_{1}=$ $\zeta_{\mathrm{r}}=0$ and $\zeta_{\mathrm{l}}=\zeta_{\mathrm{r}}=0.05$, respectively; for Figure 9.b these quantities are $c_{\mathrm{p}}=5.084 \times 10^{6}$ and $4.964 \times 10^{6} \mathrm{~kg} / \mathrm{s}$, respectively. The previous formulation [14] indicates that $\zeta_{\mathrm{A}}=0.1981$ (equation (4)) should be taken in the Kelvin-Voigt model. In the case shown in Figure 9.a, $c_{\mathrm{A}}=1.060 \times 10^{6} \mathrm{~kg} / \mathrm{s}$ and $c_{\mathrm{A}}=3.350 \times 10^{6} \mathrm{~kg} / \mathrm{s}$, for $\zeta_{\mathrm{l}}=\zeta_{\mathrm{r}}=$ 0 and $\zeta_{1}=\zeta_{\mathrm{r}}=0.05$, respectively; in the case shown in Figure 9.b, $c_{\mathrm{A}}=5.011 \times 10^{6} \mathrm{~kg} / \mathrm{s}$ (equation (3)). Therefore, there are significant differences among the damping parameter given by the existing and proposed formulations.

\subsection{Numerical results}

As discussed previously, the dynamic behavior of the colliding frames is simulated with the software SeismoStruct [33]. For comparison purposes, two types of analysis are carried out: in the first type the damping parameter of the Kelvin-Voigt model is determined according to the proposed formulation and in the second one it is set after the previous approach [14]. The time step is $\Delta t=0.0005 \mathrm{~s}$; this value has been selected mainly based on the highly short impact duration (Figure 12.b and Figure 12.d) [11]. The frames damping is described with a mass and stiffness-proportional Rayleigh formulation; $5 \%$ is chosen for the first and third modes. Given the rather moderate height of the colliding frames, the $P-\Delta$ effects are not taken into consideration. 
Figure 12 displays time histories of the impact force corresponding to dynamic analyses using the proposed formulation. Figure 12.a and Figure 12.c refer, for $\zeta_{1}=\zeta_{\mathrm{r}}=0$, to Figure 9.a and Figure 9.b, respectively. Figure 12.b and Figure 12.d contain zoom views of the strongest impacts of Figure 12.a and Figure 12.c, respectively.

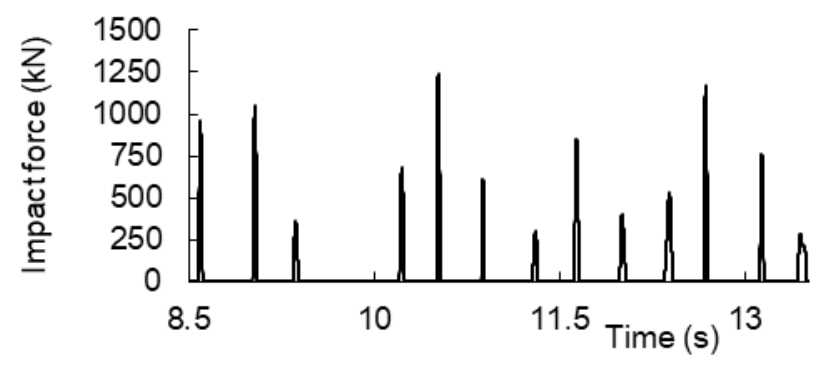

(a) Impact force time-history for 3-story 5-bay and 3story 2-bay frames

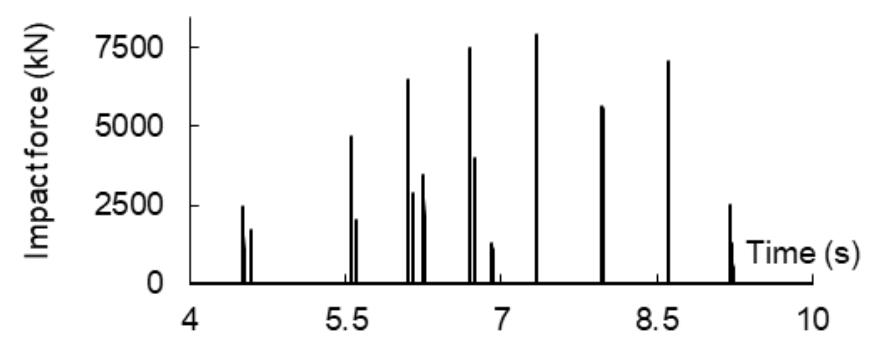

(c) Impact force for 5-story 2-bay and 3-story 2-bay frames

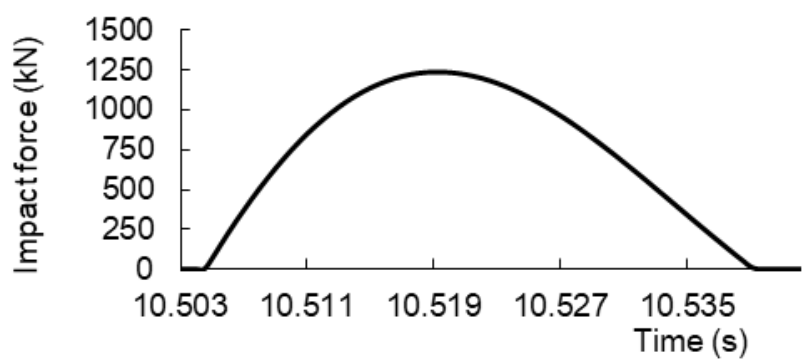

(b) Strongest impact time-history for 3-story 5-bay and 3-story 2-bay frames

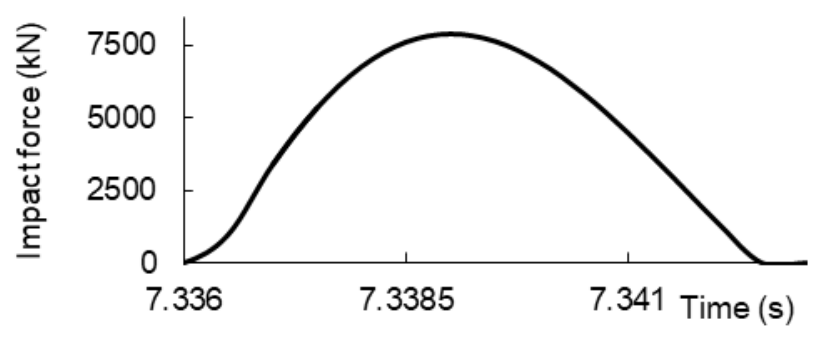

(d) Strongest impact time-history for 5-story 2-bay and 3-story 2-bay frames

Figure 12. Impact force time history for $\zeta_{1}=\zeta_{\mathrm{r}}=0$

Figure 12.a and Figure 12.c show that, as expected, the collisions are rather scarce and highly short. In Figure 12.b, the impact duration is $0.035 \mathrm{~s}$ (between instants $10.5045 \mathrm{~s}$ and $10.5395 \mathrm{~s})$ and in Figure 12.d, is $0.0065 \mathrm{~s}(7.3425-$ $7.3360 \mathrm{~s}$ ). The predicted impact durations according to the previous formulation [14] (equation (4)) for Figure 12.b and Figure 12.d are $0.0406 \mathrm{~s}$ and $0.00618 \mathrm{~s}$, respectively. The impact durations foreseen by the proposed formulation are $t_{\mathrm{imp}}=0.0378 \mathrm{~s}$ (Figure 12.b) and $t_{\mathrm{imp}}=0.00610 \mathrm{~s}$ (Figure 12.d). Figure 12.b and Figure 12.d show that the time histories of the impact forces are basically shaped as segments of damped sinusoidal waves; this is coherent with equations (1), (9) and (13).

After the conducted dynamic analyses, the values of the coefficients of restitution corresponding to the strongest impacts are determined using equation (2). Table 4 displays the obtained results.

\begin{tabular}{|c|c|c|c|c|}
\hline \multicolumn{5}{|c|}{ Table 4. Ratios $r_{\mathrm{A} /} r_{\mathrm{T}}$ and $r_{\mathrm{p}} / r_{\mathrm{T}}$ in the example simulation } \\
\hline \multirow{2}{*}{ Formulation } & 3-story 5-bay and 3-story 2-bay frames & \multicolumn{2}{|c|}{ 5-story 2-bay and 3-story 2-bay frames } \\
\cline { 2 - 5 } & $\zeta_{\mathrm{l}}=\zeta_{\mathrm{r}}=\mathbf{0}$ & $\zeta_{\mathrm{l}}=\zeta_{\mathbf{r}}=\mathbf{0 . 0 5}$ & $\zeta_{\mathrm{l}}=\zeta_{\mathrm{r}}=\mathbf{0}$ & $\zeta_{\mathrm{l}}=\zeta_{\mathrm{r}}=\mathbf{0 . 0 5}$ \\
\hline $\boldsymbol{r}_{\mathbf{A} / \boldsymbol{r}_{\mathbf{T}}}$ & 1.053 & 0.990 & 1.047 & 0.901 \\
\hline $\boldsymbol{r}_{\mathrm{p} /} \boldsymbol{r}_{\mathrm{T}}$ & 1.018 & 1.001 & 1.029 & 1.028 \\
\hline
\end{tabular}

Table 4 shows that the coefficient of restitution determined after the dynamic analyses using the proposed approach $\left(r_{\mathrm{p}}\right)$ are closer to the target factor $\left(r_{\mathrm{T}}\right)$ than those using the previous formulation $\left(r_{\mathrm{A}}\right)$ [14]; the gains range between $0.9 \%$ and $7.1 \%$. As discussed previously after Figure 6, this circumstance can be read as a more satisfactory performance of the offered algorithm.

\section{NUMERICAL SIMULATION OF TESTING ON COLLIDING BUILDINGS}

\subsection{Experiment}

This section presents the numerical simulation of a shaking-table test on seismic pounding between two reducedscale laboratory models of RC equal-height 2-story single-bay building frames [34]. Figure 13 displays a sketch of the test mockup (Figure 13.a), and the numerical model that is employed in the simulation (Figure 13.b). 


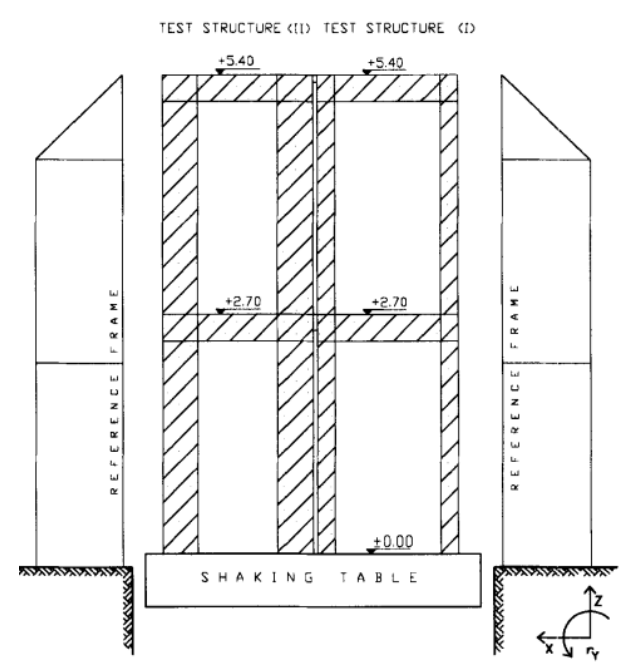

(a) Front view of the pounding frames [34]

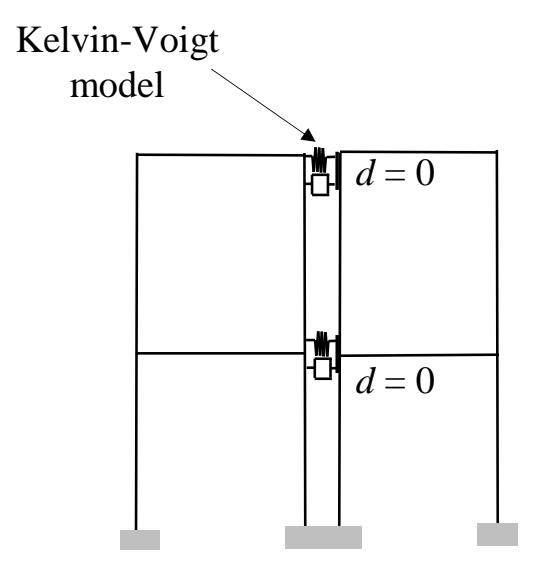

(b) Model of the pounding frames

Figure 13. Shaking table simulated experiments

The left frame was designed to be stiffer and lighter than the right one; thus, their fundamental periods were 0.139 $\mathrm{s}$ and $0.243 \mathrm{~s}$, respectively. There was no initial separation between both frames. The experiments consisted in exciting the pair of frames in two situations: without and with pounding. In both cases, the input was a harmonic wave but whose amplitude was modulated by a half-sinus wave with $12 \mathrm{~s}$ period [34]; hence, the input duration was $6 \mathrm{~s}$. The maximum amplitude was $0.08 \mathrm{~g}$ and the period of the aforementioned harmonic wave was $0.244 \mathrm{~s}$; this value was selected to generate resonance in the right (flexible) frame.

\subsection{Numerical simulation}

The experiment is simulated with SeismoStruct [33] by considering a pair of 2-D frames. The structural behavior is assumed to be linear, and the structure is discretized with elastic 2-node frame elements. The damping ratio for each frame is 0.05 .

The pounding is described with two zero-gap $(d=0)$ Kelvin-Voigt models (Figure 13.b). Their stiffness is selected equal to the axial one of the left colliding beam. The damping parameter of the Kelvin-Voigt models is selected after the Anagnostopoulos [14] and the proposed formulations. The target coefficient of restitution is $r_{\mathrm{T}}=0.527$; then, $\zeta_{\mathrm{A}}=0.2$ (equation (4)) and $\zeta_{\mathrm{p}}=0.2$ (Figure 3). Noticeably, the coincidence between $\zeta_{\mathrm{A}}$ and $\zeta_{\mathrm{p}}$ arises from the absence of seismic gap (section 3). The damping parameters $c_{\mathrm{A}}$ and $c_{\mathrm{p}}$ are obtained after equation (3) and equation (8), respectively. In the top Kelvin-Voigt model, $c_{\mathrm{A}}=5385 \mathrm{~kg} / \mathrm{s}$ and $c_{\mathrm{p}}=5796 \mathrm{~kg} / \mathrm{s}$; in the bottom model, $c_{\mathrm{A}}=$ $3440 \mathrm{~kg} / \mathrm{s}$ and $c_{\mathrm{p}}=10129 \mathrm{~kg} / \mathrm{s}$. Table 5 displays the parameters of the proposed algorithm.

\begin{tabular}{|c|c|c|c|c|c|c|c|c|c|}
\hline \multicolumn{10}{|c|}{ Table 5. Input parameters of the proposed algorithm in the experiment simulation } \\
\hline Floor No. & $\boldsymbol{m}_{\mathbf{1}}(\mathbf{k g})$ & $\boldsymbol{c}_{\mathbf{l}}(\mathbf{k g} / \mathbf{s})$ & $\boldsymbol{k}_{\mathbf{l}}(\mathbf{k N} / \mathbf{m m})$ & $\boldsymbol{\mu}$ & $\boldsymbol{\gamma}$ & $\boldsymbol{d} \mathbf{( \mathbf { m m } )}$ & $\boldsymbol{v}_{\mathbf{l}}(\mathbf{m} / \mathbf{s})$ & $\boldsymbol{v}_{\mathbf{r}}(\mathbf{m} / \mathbf{s})$ & $\boldsymbol{r}_{\mathbf{T}}$ \\
\hline $\mathbf{1}$ & 2867 & 9730 & 3.301 & 2.093 & 0.0132 & 0 & $*$ & $*$ & 0.527 \\
\hline $\mathbf{2}$ & 2669 & 5633 & 1.188 & 2.542 & 0.004 & 0 & $*$ & $*$ & 0.527 \\
\hline
\end{tabular}

(*) Since $d=0$, these values are not utilized

The time integration is performed with the Newmark algorithm using constant acceleration interpolation $(\beta=0.25$ and $\gamma=0.5$ ). Progressively reduced values of the time step are selected until obtaining similar results [11]; $\Delta t=$ $0.01,0.001$ and $0.0005 \mathrm{~s}$ are considered (Table 6).

\subsection{Numerical results}

This subsection discusses the most meaningful results of the numerical simulation, namely the calculated values of the coefficient of restitution, and the top floor relative displacement, according to the Anagnostopoulos and the proposed formulations. Table 6 displays these quantities for each value of $\Delta t$. 


\begin{tabular}{|c|c|c|c|c|c|c|}
\hline \multicolumn{7}{|c|}{ Table 6. Maximum top story displacement (mm) and coefficients of restitution } \\
\hline & \multicolumn{2}{|c|}{ Without pounding } & \multicolumn{3}{|c|}{ With pounding } & \\
\cline { 2 - 8 }$\Delta \boldsymbol{t}(\mathbf{s})$ & $\begin{array}{c}\text { Left } \\
\text { Frame } \\
\text { Exp./Num. }\end{array}$ & $\begin{array}{c}\text { Right Frame } \\
\text { Exp./Numerical }\end{array}$ & $\begin{array}{c}\text { Left Frame } \\
\text { Exp./Numerical } \\
\text { proposed/Num. } \\
\text { Anagnostopoulos }\end{array}$ & $\begin{array}{c}\text { Right Frame } \\
\text { Exp./Numerical } \\
\text { proposed/Num. } \\
\text { Anagnostopoulos }\end{array}$ & $\boldsymbol{r}_{\mathrm{p}}$ & $\boldsymbol{r}_{\mathrm{A}}$ \\
\hline 0.01 & $2.60 / 3.00$ & $25.50 / 27.24$ & $5.80 / 6.08 / 6.11$ & $12.40 / 13.90 / 16.41$ & 0.5460 & 0.4574 \\
\hline 0.001 & $2.60 / 2.97$ & $25.50 / 27.11$ & $5.80 / 5.99 / 6.16$ & $12.40 / 13.77 / 16.43$ & 0.5435 & 0.4642 \\
\hline 0.0005 & $2.60 / 2.97$ & $25.50 / 27.09$ & $5.80 / 5.99 / 6.16$ & $12.40 / 13.76 / 16.42$ & 0.5433 & 0.4616 \\
\hline
\end{tabular}

Table 6 provides the following observations:

- As previously announced, as the time step is being progressively reduced, the numerical results of the maximum top story displacements and the coefficients of restitution tend to stabilize. This remark holds for both the Anagnostopoulos and the proposed formulations.

- Comparison between the numerical and experimental results shows a satisfactory agreement.

- In the simulations with pounding, the top story displacements obtained after the proposed algorithm are closer to the experimental ones than those from the Anagnostopoulos approach.

- The coefficient of restitution calculated according to the proposed algorithm $\left(r_{\mathrm{p}}\right)$ is closer to the target value than $r_{\mathrm{A}}$.

The second observation gives some insight into the effectiveness of the proposed algorithm, but cannot be considered as a full validation procedure; regarding this issue, additional experimental research is currently in progress. The last two observations stress, in this case, the higher performance of the proposed approach.

\section{CONCLUSIONS}

The Kelvin-Voigt model is commonly used for describing seismic pounding between adjoining buildings with aligned slabs; this work proposes a new approach for estimating the damping parameter of such model. The proposed approach is based on the well-known formulation by Anagnostopoulos, although taking into consideration, during the impact duration, the effect of the stiffness and damping of the colliding buildings, and their separation. Thus, the outcome of this study is an algorithm providing the damping parameter after the estimated target coefficient of restitution. This algorithm involves two simple nested iteration loops; criteria for selecting their input parameters are provided. The accuracy of the proposed algorithm is assessed by comparing the obtained value of the coefficient of restitution with the target one; obviously, the closer, the more accurate.

The obtained results corroborate that the Anagnostopoulos approach, despite its simplicity, is efficient. Beyond such confirmation, this study also shows that the proposed algorithm is able to provide more accurate approximations of the coefficient of restitution; noticeably, in some cases, the differences between both formulations are significant. As expected, this occurs when the influence of the parameters that are not accounted in the Anagnostopoulos approach is more intense: low stiffness of the Kelvin-Voigt model, big separation among the colliding buildings, non-highly violent impact, and highly damped buildings. As well, the lower the target coefficient of restitution (i.e. plastic impact), the more important the improvement provided by the proposed formulation. Importantly, this upgrading is obtained at a small computational cost, given that the proposed algorithm needs to be calculated only one time for each analysis.

The proposed algorithm is utilized in this paper in numerical analyses of collisions between pairs of RC frames, and in simulation of shaking table pounding experiments between RC frames. In both types of situations, its performance is satisfactory and fairly superior to the one of the Anagnostopoulos approach.

\section{ACKNOWLEDGEMENTS}

This work has received financial support from Spanish Government under projects BIA2014-60093-R and CGL2015-6591.

\section{REFERENCES}

1. Rosenblueth E. (1986). The 1985 earthquake: Causes and effects in Mexico City. Concrete International. 8:23-34. 
2. EERI. (1989). Loma Prieta Earthquake. October 17, 1989: Preliminary Reconnaissance Report. Earthquake Engineering Research Institute.

3. EQE. (1994). The January 17, 1994 Northridge, California Earthquake, An EQE Summary Report Retrieved from http://www.absconsulting.com/resources/Catastrophe_Reports/1994 Northridge EQ.pdf.

4. Comartin CD, Greene M, Tubbesing SK. (1995). The Hyōgo-Ken Nanbu Earthquake: Great Hanshin Earthquake Disaster, January 17, 1995: Preliminary Reconnaissance Report. Earthquake Engineering Research.

5. Kasai K, Maison BF. (1997). Building pounding damage during the 1989 Loma Prieta earthquake. Engineering Structures, 19(3):195-207.

6. Weimin D. (2000). Chi-Chi, Taiwan Earthquake Event Report. Risk Management Solutions, 16. Retrieved from http://forms2.rms.com/rs/729-DJX-565/images/eq_chi_chi_taiwan_eq.pdf.

7. Cole GL, Dhakal RP, Turner FM. (2012). Building pounding damage observed in the 2011 Christchurch earthquake. Earthquake Engineering and Structural Dynamics, 41(5):893-913.

8. Chouw N, Hao H. (2012). Pounding damage to buildings and bridges in the 22 February 2011 Christchurch earthquake. International Journal of Protective Structures. 3(2):123-140.

9. Gautam D, Bhetwal KK, Rodrigues H, Neupane P, Sanada Y. (2015). Observed Damage Patterns on Buildings during 2015 Gorkha (Nepal) Earthquake. New Technologies for Urban Safety of Mega Cities in Asia.

10. Cole G, Dhakal R, Carr A, Bull D. (2011). An investigation of the effects of mass distribution on pounding structures. Earthquake Engineering and Structural Dynamics, 40(6):641-659.

11. Kharazian A. (2017). Analysis of seismic pounding of moderate height RC buildings with aligned slabs. Doctoral Dissertation, Technical University of Catalonia.

12. Watanabe G, Kawashima K. (2004). Numerical simulation of pounding of bridge decks. 13WCEE, Vancouver, Canada.

13. Anagnostopoulos SA. (1988). Pounding of buildings in series during earthquakes. Earthquake Engineering and Structural Dynamics, 16(3):443-456.

14. Anagnostopoulos SA. (2004). Equivalent viscous damping for modeling inelastic impacts in earthquake pounding problems. Earthquake Engineering and Structural Dynamics, 33(8):897-902.

15. Muthukumar S, DesRoches R. (2006). A Hertz contact model with non-linear damping for pounding simulation. Earthquake Engineering and Structural Dynamics, 35(7):811-828.

16. Mahmoud S, Jankowski R. (2011). Modified linear viscoelastic model of earthquake-induced structural pounding. Iranian Journal of Science and Technology, 35:51-62.

17. Komodromos P, Polycarpou PC, Papaloizou L, Phocas MC. (2007). Response of seismically isolated buildings considering poundings. Earthquake Engineering and Structural Dynamics, 36(12):1605-1622.

18. Kun Y, Li L, Hongping Z. (2009). A modified Kelvin impact model for pounding simulation of base-isolated building with adjacent structures. Earthquake Engineering and Engineering Vibration, 8(3):433-446.

19. Barros RC, Naderpour H, Khatami SM, Mortezaei A. (2013). Influence of seismic pounding on RC buildings with and without base isolation system subject to near-fault ground motions. Journal of Rehabilitation in Civil Engineering, 1(1):39-52.

20. Jankowski R. (2005). Non-linear viscoelastic modelling of earthquake-induced structural pounding. Earthquake Engineering and Structural Dynamics, 34(6):595-611.

21. Khatiwada S, Chouw N, Butterworth JW. (2014). A generic structural pounding model using numerically exact displacement proportional damping. Engineering Structures, 62:33-41.

22. Khatiwada S, Chouw N. (2014). Limitations in simulation of building pounding in earthquakes. International Journal of Protective Structures, 5(2):123-150.

23. Khatiwada S, Chouw N, Larkin T. (2013). Simulation of structural pounding with the sears impact model. $4^{\text {th }}$ ECCOMAS Thematic Conference on Computational Methods in Structural Dynamics and Earthquake Engineering.

24. Maison BF, Kasai K. (1992). Dynamics of pounding when two buildings collide. Earthquake Engineering and Structural Dynamics, 21(9):771-786.

25. Liu Y, Liu WG, Wang X, He WF, Yang QR. (2014). New equivalent linear impact model for simulation of seismic isolated structure pounding against moat wall. Shock and Vibration, 2014:151237.

26. Anagnostopoulos SA, Spiliopoulos KV. (1992). An investigation of earthquake induced pounding between adjacent buildings. Earthquake Engineering and Structural Dynamics, 21(4):289-302.

27. Guo A, Cui L, Li S, Li H. (2015). A phenomenological contact-element model considering slight non-uniform contact for pounding analysis of highway bridges under seismic excitations. Earthquake Engineering and Structural Dynamics, 44(11):1677-1695.

28. Mavronicola E, Polycarpou PC, Komodromos P. (2015). The effect of modified linear viscoelastic impact models on the pounding response of a base isolated building with adjacent structures. $5^{\text {th }}$ International Conference on Computational Methods in Structural Dynamics and Earthquake Engineering, Crete, Greece.

29. Mavronicola E, Polycarpou PC, Komodromos P. (2016). Effect of Planar Impact Modeling on the Pounding Response of Base-Isolated Buildings. Frontiers in Built Environment, doi: 10.3389/fbuil.2016.00011.

30. Mavronicola E. (2017). Nonlinear modelling considerations on the seismic response of base-isolated buildings: 2D and 3D investigations of poundings. PhD Dissertation, University of Cyprus.

31. ACI-318-11. (2011). Building Code Requirements for Structural Concrete and Commentary (318R-11). American Concrete Institute. 
32. FEMA 356. (2000). Prestandard and Commentary for the Seismic Rehabilitation of Buildings. Federal Emergency Management Agency.

33. SeismoSoft. (2017). A computer program for static and dynamic nonlinear analysis of framed structures. Available from URL www.Seismosoft.com.

34. Papadrakakis M, Mouzakis HP. (1995). Earthquake simulator testing of pounding between adjacent buildings. Earthquake Engineering and Structural Dynamics, 24(6):811-834.

\section{LIST OF SYMBOLS}

$a_{1} / a_{\mathrm{r}}$ : Initial displacement of the left/right colliding frames (Figure 4)

A: Cross-section area

b: Constant vector given by $\mathbf{b}=(-k d, k d)^{\mathrm{T}}$ (equation (5))

$c$ : Damping. Damping of Kelvin-Voigt model according to the reference [14]

$c_{\mathrm{A}} / c_{\mathrm{p}}$ : Damping of the Kelvin-Voigt model according to the Anagnostopulos/proposed formulation

$c_{1} / c_{\mathrm{r}}$ : Equivalent damping, during the impact, of the left/right models of the buildings (Figure 2)

$d$ : Gap between two adjoining colliding buildings

$E$ : Equivalent concrete elastic deformation modulus

$k$ : Stiffness of the Kelvin-Voigt model

$k_{\mathrm{l}} / k_{\mathrm{r}}$ : Equivalent stiffness, during the impact, of the left/right models of the buildings (Figure 2)

$l / r$ : Subindexes of the left/right buildings

$L$ : Length of the colliding slabs

$\mathbf{M} / \mathbf{C} / \mathbf{K}$ : Mass/damping/stiffness matrices (equation (5))

$m$ : mass of a building or frame

$m_{1} / m_{\mathrm{r}}$ : Equivalent mass of the colliding of slabs of the left/right buildings (Figure 2)

$r, r_{\mathrm{T}}$ : Coefficient of restitution, target coefficient of restitution

$r_{\mathrm{A}} / r_{\mathrm{p}}$ : Coefficient of restitution obtained after the Anagnostopoulos/proposed formulations

$t / t_{\text {imp }} / t_{\max }$ : Time/impact duration/maximum impact duration

$T_{\mathrm{i}}$ : natural period of the $i$-th mode

$m_{\mathrm{i}}^{*}$ : equivalent modal mass of the $i$-th mode

$v_{1} / v_{\mathrm{r}}$ : Traveling (absolute) velocities of left/right slabs in the beginning of the collision

$v^{\prime} / v_{r}^{\prime}$ : Traveling (absolute) velocities of left/right slabs in the end of the collision

$\mathbf{x}$ : Displacement vector given by $\mathbf{x}=\left(x_{1}, x_{\mathrm{r}}\right)^{\mathrm{T}}$ (equation (5))

$x_{1} / x_{\mathrm{r}}$ : Coordinates of the colliding of slabs of the left/right buildings

$x_{0}$ : Initial coordinate of both colliding slabs at the onset of impact

$\beta$ : Parameter of the Newmark algorithm

$\varepsilon_{0} / \varepsilon_{\mathrm{r}} / \varepsilon_{\eta}:$ Tolerance ratio/Tolerances for the outer/inner loops

$\Phi$ : Modal matrix

$\gamma$ : Ratio $k_{\mathrm{r}} / k$; parameter of the Newmark algorithm

$\eta_{1} / \eta_{2}$ : Modal coordinates of the first/second modes. $\eta=\left(\eta_{1}, \eta_{2}\right)^{T}$

$\lambda$ : Ratio $c_{\mathrm{r}} / c$

$\mu$ : Ratio $m_{1} / m_{\mathrm{r}}$

$\omega / \omega_{1} / \omega_{2}$ : Natural frequency according to [14]/Natural frequency of the first/second modes according to the proposed formulation

$\omega_{1} / \omega_{\mathrm{r}}$ : Equivalent natural frequency, during the impact, of the left/right models of the buildings (Figure 2)

$\zeta / \zeta_{1} / \zeta_{2}$ : Damping ratio according to [14]/damping ratio of the first/second modes according to the proposed formulation

$\zeta_{1} / \zeta_{\mathrm{r}}:$ Equivalent damping ratio, during the impact, of the left/right models of the buildings (Figure 2)

$\zeta_{\mathrm{A}} / \zeta_{\mathrm{p}}$ : Damping ratio of the Kelvin-Voigt model according to the Anagnostopulos/proposed formulation 